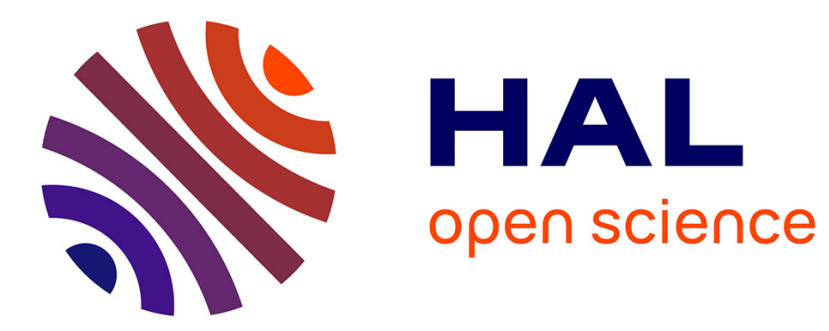

\title{
Whitham's Modulation Equations and Stability of Periodic Wave Solutions of the Korteweg-de Vries-Kuramoto-Sivashinsky Equation
}

Pascal Noble, Luis Miguel Miguel Rodrigues

\section{- To cite this version:}

Pascal Noble, Luis Miguel Miguel Rodrigues. Whitham's Modulation Equations and Stability of Periodic Wave Solutions of the Korteweg-de Vries-Kuramoto-Sivashinsky Equation. Indiana University Mathematics Journal, 2013, 62 (3), pp.753-783. hal-00943713

\section{HAL Id: hal-00943713 https://hal.science/hal-00943713}

Submitted on 8 Feb 2014

HAL is a multi-disciplinary open access archive for the deposit and dissemination of scientific research documents, whether they are published or not. The documents may come from teaching and research institutions in France or abroad, or from public or private research centers.
L'archive ouverte pluridisciplinaire $\mathbf{H A L}$, est destinée au dépôt et à la diffusion de documents scientifiques de niveau recherche, publiés ou non, émanant des établissements d'enseignement et de recherche français ou étrangers, des laboratoires publics ou privés. 


\title{
Whitham's Modulation Equations and Stability of Periodic Wave Solutions of the Korteweg-de Vries-Kuramoto-Sivashinsky Equation
}

\author{
Pascal Noble* ${ }^{*}$ L.Miguel Rodrigues ${ }^{\dagger}$
}

Keywords: modulation; periodic traveling waves; Kuramoto-Sivashinsky equations, Korteweg-de Vries equations; Bloch decomposition.

2000 MR Subject Classification: 35Q53, 35B10, 35B35, 35P10.

\begin{abstract}
We study the spectral stability of periodic wave trains of the Korteweg-de VriesKuramoto-Sivashinsky equation which are, among many other applications, often used to describe the evolution of a thin liquid film flowing down an inclined ramp. More precisely, we show that the formal slow modulation approximation resulting in the Whitham system accurately describes the spectral stability to side-band perturbations. Here, we use a direct Bloch expansion method and spectral perturbation analysis instead of Evans function computations. We first establish, in our context, the now usual connection between first order expansion of eigenvalues bifurcating from the origin (both eigenvalue 0 and Floquet parameter 0) and the first order Whitham's modulation system: the hyperbolicity of such a system provides a necessary condition of spectral stability. Under a condition of strict hyperbolicity, we show that eigenvalues are indeed analytic in the neighborhood of the origin and that their expansion up to second order is connected to a viscous correction of the Whitham's equations. This, in turn, provides new stability criteria. Finally, we study the Korteweg-de Vries limit: in this case the domain of validity of the previous expansion shrinks to nothing and a new modulation theory is needed. The new modulation system consists in the Korteweg-de Vries modulation equations supplemented with a source term: relaxation limit in such a system provides in turn some stability criteria.
\end{abstract}

\footnotetext{
*Université de Lyon, UMR CNRS 5208, Université Lyon I, Institut Camille Jordan, 43 bd du 11 novembre 1918, F-69622 Villeurbanne Cedex, France; noble@math.univ-lyon1.fr: Research of P.N. is partially supported by the French ANR Project no. ANR-09-JCJC-0103-01.

${ }^{\dagger}$ Université de Lyon, UMR CNRS 5208, Université Lyon 1, Institut Camille Jordan, 43 bd du 11 novembre 1918, F-69622 Villeurbanne Cedex, France; rodrigues@math.univ-lyon1.fr.
} 


\section{Introduction}

Coherent structures such as solitary waves, fronts or periodic traveling waves, usually play an essential role as elementary processes in nonlinear phenomena. It is both usual and useful to try first to analyze the behavior of these elementary structures with canonical models for pattern formation [8]. Here we try for such a canonical equation to relate side-band stability of periodic traveling waves with modulation averaged equations.

We focus our attention on a scalar equation of the form

$$
\partial_{t} u+6 u \partial_{x} u+\delta_{1} \partial_{x}^{2} u+\delta_{2} \partial_{x}^{3} u+\delta_{3} \partial_{x}^{4} u=0, \quad(x, t) \in \mathbb{R} \times \mathbb{R}_{+}^{*},
$$

where $\delta_{1}, \delta_{2}$ and $\delta_{3}$ are some constant real numbers. This kind of equations arises in many situations as a simplified asymptotic equation. For this purpose, when $\delta_{1}<0$, it is often sufficient to set $\delta_{2}=\delta_{3}=0$, that is to consider a viscous Burgers' equation. In the limit case $\delta_{1}=0$, if $\delta_{2} \neq 0$, for some purposes, one may also set $\delta_{3}=0$ and work with the Korteweg-de Vries equation $(\mathrm{KdV})$. However, for well-posedness issues, when $\delta_{1}<0$, one can not stop before a fourth order term ${ }^{1}$, and stop there only if $\delta_{3}>0$. This is this latter case we are interested in here. Therefore the equation we study incorporates nonlinearity, dispersion, and, as far as it is considered about constant states, dissipative instability with respect to low frequency perturbations, stability to high frequencies.

We now perform some scaling transformations to make the structure of the equation clearer. First, up to changing $(x, u)$ into $(-x,-u)$, may be assumed $\delta_{2} \geq 0$. Then if $\delta_{1}, \delta_{2}$ and $\delta_{3}$ are positive, up to changes $t=\delta_{2}\left(\delta_{1} / \delta_{3}\right)^{3 / 2} t, x=\left(\delta_{1} / \delta_{3}\right)^{1 / 2} x, u=\delta_{2}^{-1}\left(\delta_{1} / \delta_{3}\right)^{-1} u$, $\delta=\delta_{2}^{-1}\left(\delta_{1} / \delta_{3}\right)^{-1 / 2} \delta_{1}$, the above equation may be recasted into

$$
\partial_{t} u+6 u \partial_{x} u+\partial_{x}^{3} u+\delta\left(\partial_{x}^{2} u+\partial_{x}^{4} u\right)=0
$$

Note that we have ruled out the relevant case $\delta_{2}=0, \delta_{1}>0, \delta_{3}>0$, corresponding to the Kuramoto-Sivashinsky equation. Yet, in reference to this equation, we call equation (I.I) the Korteweg-de Vries-Kuramoto-Sivashinsky equation (KdV-KS). The above scaling was intended to enlighten the crucial role of the above $\delta_{1}$ parameter. Moreover, with pattern formation in mind, we have also ensured that, about any constant state, the linear most unstable mode has frequency \pm 1 . Setting $\delta=0$, the most common form of the Kortewegde Vries is recovered ${ }^{2}$. It should be clear now that the equation enters naturally in the description of weakly-nonlinear large-scale waves above a threshold where all constant states become unstable to low-frequency perturbations, threshold corresponding to $\delta=0$.

Though the Kuramoto-Sivashinsky equation has been first derived to study chemical reactions and flame stability $[17,18,22,23]$ and equation (GKS has been widely used to describe plasma instabilities, flame front propagation, turbulence in reaction-diffusion systems, for a short time we now specialize the discussion to the evolution of nonlinear waves in fluid mechanics, reflecting personal interest of the authors. A

\footnotetext{
${ }^{1}$ In some situations one may argue then that a term $D \partial_{x}^{2}\left(u^{2}\right)$ should be taken into account $[1]$, but we disregard this term here.

${ }^{2}$ That is the reason why we have chosen not to eliminate the 6 factor.
} 
detailed description of these applications may be found in $[\mathrm{CD}]$, we only say a few words about the threshold of stability. When analyzing, with free-surface incompressible Navier-Stokes equations, the evolution of a thin fluid flow down an incline plane of a given slope, appears a critical Reynolds number above which flows parallel to the incline become unstable. Above but close to this critical Reynolds number, equation (位. $)$ may be used to describe the dynamics with $\delta$ being proportional to the deviation of the Reynolds number from its critical value $\left.\frac{12}{26}\right]$. When the same shallow-flow situation is modeled rather with the SaintVenant equations, $\delta$ then corresponds to the deviation of the Froude number from its critical value [27]. We remark that the classic Kuramoto-Sivashinsky equation, not considered here, would rather correspond to modeling of a vertical film flow $[24,6]$.

Related to the instability of constant states is the fact that, for any fixed $\delta>0$, from constant states bifurcates a family of periodic traveling waves through a Hopf bifurcation. This family is enclosed by a family of solitary waves. See [7, 2] for a detailed description of phase portraits. ${ }^{3}$ Since, by nature, all constant states are spectrally unstable, all solitary waves have unstable essential spectrum and small and large periodic waves are expected to be unstable. Yet it does not forbid a priori periodic waves of intermediate periods or

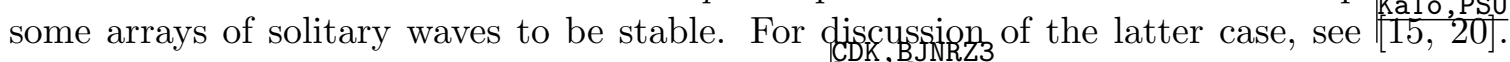
As for the former, note that numerical studies [7, 2] indeed show that for any $\delta$, even up to the Kuramoto-Sivashinsky equation, in parameter space a full band of spectrally stable periodic wave trains does exist. Note also that under precise assumptions of diffusive spectral stabilitvone may go from spectral stability to ponlinear stability under localized perturbation [2] (see also announcement of this work in [3]).

To be more precise, let us say that the spectral stability we discussed above is under arbitrary bounded perturbations. Being related to co-periodic stability, the stability we discuss in the rest of this work - side-band stability - is of weaker nature. Many numerical works and even experiments have been focused on the even weaker requirement of stability under co-periodic perturbations. Yet, to be fully significant from a realistic point of view, these studies should be at least extended to an analysis of stability under close-to-co-periodic perturbations $^{4}$, or in words of the analysis below by an analysis of the low-Floquet stability (see definition of the Bloch transform in (2.6), where the Floquet parameter is $\xi$ ). This is the well-known issue of side-band stability/instability. Determining side-band stability should tell for which waves stability to co-periodic perturbations may be observed, whereas side-band instability yields in any case spectral instability. Obviously a side-band analysis is required only where co-periodic spectrum intersects the imaginary axis, thus only when co-periodic stability is marginal, but due to translational and Galilean invariance the former intersection is always non empty, 0 being an eigenvalue of algebraic multiplicity larger than

\footnotetext{
${ }^{3}$ We note, by the way, that our analysis is intended to deal with the family of periodic wave trains that persist for all values of $\delta$ and not the ones that exist close enough to the Kuramoto-Sivashinsky equation, that is for $\delta$ large enough.

${ }^{4}$ That is perturbations given, in a co-moving frame making the wave both stationary and periodic of period one, by $x \mapsto e^{i \xi x} \tilde{u}(x)$ with $\tilde{u}$ co-periodic, square-integrable on $[0,1]$ and $\xi$ small. Recall that Floquet theory tells that, relaxing the smallness of $\xi$ to $\xi \in[-\pi, \pi]$, the full spectrum is attained with such perturbations. We call $\xi$ a Floquet or a Bloch parameter.
} 
or equal to 2 .

The present work is restricted to the discussion of this side-band stability. Actually the role of dynamics of small Floquet perturbations is far greater than the one of deciding robustness of co-periodic predictions. For spectrally stable waves, it is expected and observed that the full nonlinear dynamic is driven by the evolution of the small Floquet part of the solution. The reason for this is that it is expected that for stable waves the marginally stable part of the spectrum corresponds to the above mentioned modes, a twodimensional space of co-periodic perturbations generated from translational and Galilean invariance. It is thus of prominent importance to understand how this space perturbs for low Floquet parameters. More generally, for spectrally stable periodic traveling waves, the general aim is, thanks to a suitable parameterization of close-by periodic traveling waves, to reduce at leading-order the nonlinear long-time dynamic about a periodic traveling wave to the nonlinear dynamic of parameters about a constant state. Hence the importance of phase dynamics [9] (when only one parameter is involved) or more generally of modulation dynamics [25]. Note that the averaging modulation process associates low Floquet modes of the full solution to low Fourier modes of parameters. Therefore one expects for stable waves the long-time dynamic to be of slow modulation type, that is, in some local scale, behavior of periodic traveling wave type but with parameters evolving on larger scales.

The purpose of deriving modulation averaged systems, that we call Whitham's systems, is to propose approximate equations for the evolution of the parameters. The type of the modulation system depends then on the desired degree of accuracy. Note that the dimension of the system is not naively deduced from the dimension of the equation but is the one of the family of periodic traveling waves (considered up to translation invariance). In our case, we will assume the generic situation where the family of periodic traveling is parameterized by phase shift, wave number $k$ and mean $M$ (see assumption (A') below), so that we are looking for a system describing the slow evolution of $(k, M)$.

Our goal is not to decide side-band stability but to relate it to properties of some modulation averaged systems, and, when side-band stability is met, to elucidate where one can read both sets of parameters that are crucial for the description of the evolution of $(k, M)$ : linear group velocities and diffusion coefficients. For this purpose, we derive on a formal level two kinds of modulation systems, at leading-order a system involving only first-order derivatives of $(k, M)$, then a second-order correction of the previous system. We then validate these systems at the spectral level. We prove that the hyperbolicity of the first-order Whitham's system is a necessary condition for side-band stability, and that, when hyperbolicity is met, Fourier eigenvalues of the system yield linear group velocities. Assuming strict hyperbolicity of the first-order system, we then prove that second-order expansions of lowFourier eigenvalues of the second-order system yield second-order expansions of low-Floquet eigenvalues of the original system. This means that stability with respect to low-frequency perturbations in the second-order modulation system ${ }^{5}$ is a necessary condition for side-band

\footnotetext{
${ }^{5}$ On a formal level, a priori many modulation averaged systems with possibly different high-frequency behaviors may be derived to match the slow modulation evolution; hence the relevance for the original system of only a low-frequency Kawashima-type condition: low-frequency hyperbolic modes should be viscously
} 
stability of the original system, and when it is fulfilled it comes with the desired diffusion coefficients. Moreover, when both hyperbolicity and diffusion conditions are satisfied in a strict sense, they imply side-band stability. To tell it in another short way : when taken in a strong sense, co-periodic stability plus modulational stability yield side-band stability.

The role of hyperbolicity of the first-order Whitham's system is now well-known [21]. Yet, instead of relying on the Evans function calculation [21], we reprove it here by first showing regularity of critical eigenmodes with respect to Floquet parameter and then directly inspecting low-Floquet expansions. Main advantages are that we can easily rise the order of approximation and that it also provides in a natural way relation among eigenfunctions (not only eigenvalues). Although we do not address this question here but rather postpone it to further work, recall that a final goal is to prove that the full nonlinear evolution may indeed be "reduced" to the evolution of the second-order system mentioned above. For this purpose, description of eigenfunctions is crucial.

In the limit $\delta$ goes to zero ${ }^{6}$, the above scenario yields poor stability results. Indeed a close inspection reveals that the fixed- $\delta$ low-Floquet expansions we discussed actually involve not only $\xi$ but also $\xi / \delta$ so that the low-Floquet stability mentioned above should be read in the $\delta \rightarrow 0$ limit as stability to perturbations corresponding to Floquet parameters $\xi$ such that $|\xi| \leq c \min (\{1, \delta\})$, with $c$ a small fixed constant. But, elaborating about the Korteweg-de Vries equation, there is room for improvement in this small- $\delta$ limit. In particular, from

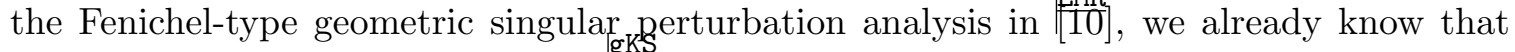
profiles of periodic wave trains of (I.I) perturb for small $\delta$, in a regular way, from some wave trains of the $\mathrm{KdV}$ equation. To elucidate how the ratio $\delta / \xi$ enters in the way the small Floquet limit $\xi \rightarrow 0$ interplays with the $\mathrm{KdV}$ limit $\delta \rightarrow 0$, we perform a modulation procedure where this ratio is fixed. This is materialized by setting $\delta=\varepsilon \bar{\delta}$, where $\bar{\delta}$ is some fixed nonnegative number and $\varepsilon$ is the inverse of the characteristic scale on which evolves local parameters, that is $\varepsilon_{\widetilde{\mathrm{KS}}}|\xi|$ where $\xi$ is a Fourier parameter for the Whitham's system,

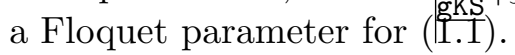

The new modulation procedure leads to a system of dimension three (and not two), reflecting the dimension of the family of periodic traveling waves for $(\mathrm{KdV})$. Not all periodic wave trains of $(\mathrm{KdV})$ provide traveling waves of (1.1). Indeed a selection criterion rules out one dimension. Though we derived it in a slightly different way, when $\bar{\delta}=0$ we recover the first-order Whitham's system of $(\mathrm{KdV})$ [25]. This system is for $\bar{\delta} \neq 0$ modified by a relaxation term of the form $\bar{\delta} \times$ (selection criterion). Constant solutions of the full Whitham's system corresponds to wave trains of (KdV) actually generating periodic waves for (1.1). Moreover, the relaxation limit $\bar{\delta} \rightarrow \infty$ limit in the Whitham's system yields back the limit $\delta \rightarrow 0$ of the Whitham's system of the fixed- $\delta$ modulation.

The relaxation structure suggests that the natural conditions yielding side-band stability for small $\delta$ are:

- hyperbolicity of the Whitham's system for (KdV);

damped by the second-order part of the modulation system.

${ }^{6}$ Recall the zone $\delta$ small is precisely the region we are most interested in. 
- dissipative nature of the relaxation term;

- hyperbolicity of the limit $\delta \rightarrow 0$ of the Whitham's system of (IT.1);

- intertwining of linear group velocities.

We formulate these subcharacteristic conditions in a precise way in the dedicated section. Yet we do not enter into a precise analysis of their role. The first reason for this is that it is not clear to us whether or not a full coherent expansion may be obtained in a full region $(\xi, \delta)$ small, and it would be very unsatisfactory to discuss a result for moderate $\delta / \xi$. The second reason is that, nevertheless, we hope in a further work, to combine a detailed low-Floquet analysis, with regular expansions of moderate-Floquet, and energy estimates for high-Floquet ${ }^{7}$ in order to prove, for small $\delta$, a full diffusive spectral stability for a band of periodic traveling waves. These subcharacteristic conditions should play a key-role in the low-Floquet analysis, yet the present discussion does not reveal in which precise sense.

The organization of the rest of the paper follows the lines of the previous discussion. In section 2 , we state the main assumptions under which the fixed- $\delta$ analysis holds. In particular, we describe how periodic wave trains of $(\mathrm{KdV}-\mathrm{KS})$ are parameterized. In section 3 , keeping $\delta$ fixed, we discuss side-band stability and modulation averaged equations. There we examine the regularity of eigenmodes with respect to Floquet parameter $\xi$ and perform expansions with respect to this parameter, then derive the Whitham's modulation systems and show that modulation equations linearized about fixed parameters provide the correct expansion of critical eigenvalues, up to order one for the first-order Whitham's system and up to second order for the second-order modulation system. In section 4 , to go beyond, in the $\mathrm{KdV}$ limit $\delta \rightarrow 0$, the restriction $\xi / \delta$ small, we introduce another set of modulation equations, which turns out to be the Whitham's modulation system for (KdV) supplemented by a source term of order $\bar{\delta} \sim \delta /|\xi|$ and discuss for this system the subcharacteristic conditions deduced from relaxation theory for hyperbolic systems.

\section{$2 \quad$ Preliminaries}

\section{Parameterization}

We start discussing parameterization of periodic traveling waves. The final form of the latter will wait until the end of the section.

We first look for periodic traveling waves $u$ of (IKS), with speed $c$ and period $X$, as $u(x, t)=U(x-c t)$, where $U$ is a $X$-periodic function that satisfies

$$
-c U^{\prime}+6 U U^{\prime}+U^{\prime \prime \prime}+\delta\left(U^{\prime \prime}+U^{\prime \prime \prime \prime}\right)=0 .
$$

Integrating once introduces a constant of integration $q$ and turns ( $\left(\mathrm{Ep}_{2.1}^{\mathrm{p}}\right)$ into

$$
-c U+3 U^{2}+U^{\prime \prime}+\delta\left(U^{\prime}+U^{\prime \prime \prime}\right)=q .
$$

\footnotetext{
${ }^{7}$ Of course $\xi \in[-\pi, \pi]$ is bounded, but high should be understood as high in comparison with $\delta$.
} 
Denote $U(\cdot ; b, c, q)$ the maximal solution of $\left(\frac{\text { ep I I }}{2.2}\right)$ such that $\left(U, U^{\prime}, U^{\prime \prime}\right)(0 ; b, c, q)=b$, and $O_{c, q, X}$ the open set of $b$ s such that $U(\cdot ; b, c, q)$ is defined on $[0, X]$. We may then define $D=\bigcup_{c, q, X} O_{c, q, X} \times\{(c, q, X)\}$ and on this open subset of $\mathbb{R}^{3} \times \mathbb{R} \times \mathbb{R} \times \mathbb{R}$ the Poincaré return map $H$ as

$$
\begin{aligned}
& H: \quad D \quad \rightarrow \mathbb{R}^{3} \\
& (b, c, q, X) \mapsto\left(U, U^{\prime}, U^{\prime \prime}\right)(X ; b, c, q)-b .
\end{aligned}
$$

With a slight abuse of notations, $H^{-1}(\{0\})$ represents the set of all periodic traveling waves of (商. . Fixing $(\bar{b}, \bar{c}, \bar{q}, \bar{X}) \in H^{-1}(\{0\})$, corresponding to some nontrivial wave, we make the following assumption on the (local) structure of $H^{-1}(\{0\})$.

(A) The map $H$ is full rank at $(\bar{b}, \bar{c}, \bar{q}, \bar{X})$.

By the Implicit Function Theorem and translational invariance, condition (A) implies that the set of periodic solutions in the vicinity of the $\bar{X}$-periodic function $\bar{U}=U(\cdot ; \bar{b}, \bar{c}, \bar{q})$ forms a smooth 3-dimensional manifold

$$
\{(x, t) \mapsto U(x-\alpha-c(\beta) t ; \beta)\} \mid(\alpha, \beta) \in \mathbb{R} \times O\} \text { with } O \text { an open subset of } \mathbb{R}^{2} .
$$

The question of the parameterization of periodic wave trains is important here in order to derive modulation equations. In view of $(2.2)$, one could wish to look for $(X, q)$ or $(c, q)$ as natural parameters for periodic wave trains. However, first, these parameterizations may not be admissible, and, second, from an averaging point of view, $(X, M)$ (or $(k, M)$ with $k$ a wavenumber), where $M=\langle U\rangle=X^{-1} \int_{0}^{X} U(x) \mathrm{dx}$ is the mean value, seems a more adequate choice. Moreover, for the present profile equation $\left(\frac{\mathrm{ep}}{2.1}\right)$, Galilean invariance makes parameterization by $M$ easier. If $(U, c)$ is a solution, so is $(U-M, c-6 M)$ for any $M \in \mathbb{R}$, so that in some sense as far as only parameterization (and not spectral properties) is concerned the mean value may be factored out. As a consequence, one may search first for periodic wave trains of $\left(\frac{\mathrm{ep}}{2.1}\right)$ satisfying the additional constraint $\langle U\rangle=X^{-1} \int_{0}^{X} U(x) \mathrm{dx}=0$. Denoting $U^{(0)}(\cdot ; k), c^{(0)}(k)$ a mean-free solution of $(2.1)$, with period $X=k^{-1}$, one recovers (locally in parameter space and up to translations) the full family of periodic wave trains, now denoted $U(\cdot ; k, M), c(k, M))$, by setting

$$
U(\cdot ; k, M)=M+U^{(0)}(\cdot ; k), \quad c(k, M)=6 M+c^{(0)}(k) .
$$

To make the parameterization of practical use (in particular in combination with the Bloch transform), we still have to normalize wave profiles periods to one so that, although we do not modify names, $U(\cdot ; k, M)_{S}$ will now denote a periodic function of period 1 and mean $M$ such that a solution to (1.1) is given by $u(x, t)=U(k(x-c(k, M) t) ; k, M)$. For later references, let us write explicitly that $U(\cdot ; k, M)$ is thus solution, of period 1 , of

$$
k\left(3 U^{2}-c(k, M) U\right)^{\prime}+k^{3} U^{\prime \prime \prime}+\delta\left(k^{2} U^{\prime \prime}+k^{4} U^{\prime \prime \prime \prime}\right)=0, \quad\langle U\rangle=M
$$

where $\langle U\rangle=\int_{0}^{1} U$.

For the discussed reasons, we strengthen (A) into

(A') The map $H$ is full rank at $(\bar{b}, \bar{c}, \bar{q}, \bar{X})$ and a parameterization by $(k, M)$ is admissible. 


\section{Bloch transform}

We will not need much about the Bloch transform but it underlies our spectral expansions. The Bloch transform rewrite Fourier transform in view of Floquet theory. It groups together Fourier modes corresponding to the same Floquet parameter. Explicitly, the Bloch transform $\check{g}$ of a Schwartz-class function $g$ is given by

checkg

$$
\check{g}(\xi, x):=\sum_{j \in \mathbb{Z}} \hat{g}(\xi+2 j \pi) e^{i 2 \pi j x},
$$

where $\hat{g}(\xi):=\frac{1}{2 \pi} \int_{\mathbb{R}} e^{-i \xi x} g(x) \mathrm{dx}$ is the Fourier transform of $g$. The Bloch transform comes with an inverse formula

Brep

$$
g(x)=\int_{-\pi}^{\pi} e^{i \xi x} \check{g}(\xi, x) \mathrm{d} \xi
$$

and a Parseval equality $\|g\|_{L^{2}(\mathbb{R})}=(2 \pi)^{1 / 2}\|\check{g}\|_{L^{2}([-\pi, \pi] \times[0,1])}$. A consequence is that we may write any $g \in L^{2}(\mathbb{R})$ as a "superposition" of functions $x \mapsto e^{i \xi x} \check{g}(\xi, x)$ with $\check{g}(\xi, \cdot)$ of period 1. This transform is well-behaved with respect to differential operator with periodic coefficients.

Let $L$ be the linear operator arising form the linearization of (IKS $)$ about a wave $\bar{U}$, in a co-moving frame $(\bar{k}(x-\bar{c} t), t): \partial_{t} v=L[v]$. Thus $L$ is the differential operator with coefficients of period 1 ,

$$
L[g]=-\bar{k}((6 \bar{U}-\bar{c}) g)^{\prime}-\bar{k}^{3} g^{\prime \prime \prime}-\delta\left(\bar{k}^{2} g^{\prime \prime}+\bar{k}^{4} g^{\prime \prime \prime \prime}\right) .
$$

The Bloch transform "diagonalizes" $L$ in the sense that

$$
(L g)(x)=\int_{-\pi}^{\pi} e^{i \xi x}\left(L_{\xi} \check{g}(\xi, \cdot)\right)(x) \mathrm{d} \xi
$$

i.e. $(\check{L} g)(\xi, x)=\left(L_{\xi} \check{g}(\xi, \cdot)\right)(x)$, where $L_{\xi}$ are differential operators, with coefficients of period 1 , acting on periodic functions via

$$
\left(L_{\xi} w\right)(x):=e^{-i \xi x} L\left[e^{i \xi \cdot} w(\cdot)\right](x) .
$$

Thus the Bloch transform reduces $L$ to operations on co-periodic functions.

Since the Bloch operator-valued symbols $L_{\xi}$ are relatively compact perturbations of the same operator $L_{0}$ with domain $H_{\text {per }}^{4}([0,1])$ compactly embedded in $L_{\text {per }}^{2}([0,1])$, their spectrum consists entirely of discrete eigenvalues which, furthermore, depend continuously on the Bloch parameter $\xi$. Then, by standard considerations relying on inverse formula and Parseval identity, follows

$$
\sigma_{L^{2}(\mathbb{R})}(L)=\bigcup_{\xi \in[-\pi, \pi)} \sigma_{L_{\mathrm{per}}^{2}([0,1])}\left(L_{\xi}\right)
$$

see [12] for details. As a result, the spectrum of $L$, which consists entirely of essential spectrum, may be decomposed into countably many continuous curves $\lambda(\cdot)$ such that, for $\xi \in[-\pi, \pi), \lambda(\xi)$ is an eigenvalue of $L_{\xi}$. 


\section{Structure of the kernel}

In what follows, we focus only on low Bloch numbers. As a starting point, we have to determine the spectrum of $L_{0}$. The purpose of the following lemma is to decide what can be deduced from assumption (A').

NL0 Lemma 2.1. The following assertions are equivalent.

1. Assumption ( $\left.A^{\prime}\right)$ is fulfilled.

2. The value 0 is an eigenvalue of $L_{0}$ of geometric multiplicity 1 and algebraic multiplicity 2. Moreover, the generalized 0-eigenspace is determined by

$$
\bar{U}^{\prime} \in \operatorname{Ker} L_{0}, \quad 1 \in \operatorname{Ker}\left(L_{0}^{2}\right) \backslash \operatorname{Ker}\left(L_{0}\right), \quad 1 \in \operatorname{Ker}\left(L_{0}^{*}\right)
$$

where $L_{0}^{*}$ denotes the formal adjoint of $L_{0}$.

One should not be confused by the fact that constant functions appears in two different places for two different reasons. The relation to the adjoint stems from the fact that we deal with a conservation law. The other one is related to $\partial_{M} \bar{U}=1$.

As a preliminary remark, note that from the fact that $L_{0}$ have compact resolvents one deduces that $L_{0}$ is a Fredholm operator ${ }^{8}$ of index 0 and that for any positive integer $k$ dimensions of $\operatorname{Ker}\left(L_{0}^{k}\right)$ and $\operatorname{Ker}\left(\left(L_{0}^{*}\right)^{k}\right)$ coincide.

Proof. Let us first assume a $(k, M)$ parameterization. From translational invariance one readily deduces (differentiating the profile equation with respect to the $\alpha$ of $(2.4)$, having in mind that $c$ and $k$ do not depend on $\alpha$ ) that $\bar{U}^{\prime}$ lies in the null space of $L_{0}$. We claim $\operatorname{Ker}\left(L_{0}\right)=\operatorname{span}\left(\bar{U}^{\prime}\right)$.

Let $\varphi$ be a 1-periodic function satisfying $L_{0} \varphi=0$. The equation yields that $\varphi$ is necessarily smooth. Then there is a $q_{\varphi}$ such that, with obvious notations, $\mathrm{d} H\left(\bar{U}(0), \bar{U}^{\prime}(0), \bar{U}^{\prime \prime}(0), \bar{c}, \bar{q}, \bar{X}\right)$. $\left(\varphi(0), \varphi^{\prime}(0), \varphi^{\prime \prime}(0), 0, q_{\varphi}, 0\right)=(0,0,0)$. This means that there exist $\left(\alpha_{\varphi}, k_{\varphi}, M_{\varphi}\right)$ such that

$$
\left\{\begin{array}{rl}
\varphi & =\alpha_{\varphi} \bar{U}^{\prime}+k_{\varphi} \partial_{k} \bar{U}+M_{\varphi} \partial_{M} \bar{U} \\
0 & =k_{\varphi} \partial_{k} \bar{c}+M_{\varphi} \partial_{M} \bar{c} \\
q_{\varphi} & =k_{\varphi} \partial_{k} \bar{q}+M_{\varphi} \partial_{M} \bar{q} \\
0 & =-k_{\varphi} \frac{1}{\bar{k}^{2}}
\end{array} .\right.
$$

Thus $k_{\varphi}=0$. However, differentiating the profile equation with respect to $M$ yields

$$
L_{0}\left[\partial_{M} \bar{U}\right]=-\bar{k} \partial_{M} \bar{c} \bar{U}^{\prime} .
$$

Since $\partial_{M} c=6$ and the wave is nontrivial, this gives $M_{\varphi}=0$. Our claim is proved.

Next, since $\partial_{M} \bar{U}=1$, we have already proved $L_{0}[1]=-6 \bar{k} \bar{U}^{\prime}$. Thus 0 is necessarily an eigenvalue of algebraic multiplicity larger than 1 . To see that it is exactly of algebraic

\footnotetext{
${ }^{8}$ When considered as a bounded operator defined on its domain, as is usual for unbounded operators.
} 
multiplicity 2, it remains to prove that 1 does not belong to the range of $L_{0}$. Yet, since (I.I) is a conservation law, the constant function 1 lies in the kernel of $L_{0}^{*}$. Of course $\langle 1,1\rangle_{L^{2}([0,1])}=1 \neq 0$, hence the result. Note that even for equations for which $\partial_{M} \bar{U}$ is not explicitly known, still would stand $\left\langle 1, \partial_{M} \bar{U}\right\rangle_{L^{2}([0,1])}=\partial_{M} \bar{M}=1$.

Now let us assume the announced structure of the kernels. This assumption implies that the kernel of $\mathrm{d} H\left(\bar{U}(0), \bar{U}^{\prime}(0), \bar{U}^{\prime \prime}(0), \bar{c}, \bar{q}, \bar{X}\right)_{\mid \mathbb{R}^{3} \times\{0\} \times \mathbb{R} \times\{0\}}$ is of dimension at most 1 and thus its range is of dimension at least 3 This gives assumption (A) but also the possibility of a parameterization of type $\left(\frac{e: \text { manifold }}{2.4) \text { with }} \beta=(c, X)\right.$ hence also with $\beta=(c, k)$. Now just observe that with this parameterization $\partial_{c} M=1 / 6 \neq 0$. A more robust argument involving Evans function computations may also replace this simple observation. This completes the proof.

Mark that in the previous proof, scalar products always refer to the canonical scalar product on $L_{\text {per }}^{2}([0,1])$. From now on it will be denoted by $\langle\cdot, \cdot\rangle$.

\section{Modulational Stability}

We inspect now how the eigenvalues of $L_{\xi}$ may bifurcate from $(\lambda, \xi)=(0,0)$ and relate this with Whitham's averaged equations for small-Floquet perturbations of periodic wave trains. We first prove the regularity of the two eigenvalues bifurcating from the origin $(\lambda, \xi)=(0,0)$ and compute an expansion up to order 2 with respect to $\xi$. Then, we show that, up to first order, they are given by the dispersion relations of classical Whitham's modulations equations and, up to second order, related to a viscous correction of Whitham's equations.

\section{Regularity}

Recall that assumption (A') induces the presence of a Jordan block at $\lambda=0$ for the operator $L_{0}$. A careful analysis of the regularity is thus needed. This is the purpose of the next lemma, inspired by a corresponding result in $[13]$.

Lemma 3.1. Assume ( $\left.A^{\prime}\right)$.

Then there exist $\left.\xi_{0} \in\right] 0, \pi\left[, \varepsilon_{0}>0\right.$ and two curves, $\left.j=1,2, \lambda_{j}:\right]-\xi_{0}, \xi_{0}\left[\rightarrow B\left(0, \varepsilon_{0}\right)\right.$ such that, when $|\xi|<\xi_{0}$,

$$
\sigma\left(L_{\xi}\right) \cap B\left(0, \varepsilon_{0}\right)=\left\{\lambda_{1}(\xi), \lambda_{2}(\xi)\right\} .
$$

Moreover these two critical curves are differentiable at 0 . Thus they expand as

$$
\lambda_{j}(\xi) \stackrel{0}{=} i \bar{k} \xi \lambda_{0, j}+o(\xi), \quad j=1,2 .
$$

Assume moreover

(B) $\lambda_{0,1} \neq \lambda_{0,2}$. 
Then the curves $\lambda_{j}$ are analytic in a neighborhood of 0 . Up to a change of $\xi_{0}$, there exist, for $0<|\xi|<\xi_{0}$, dual right and left eigenfunctions $\left(q_{j}(\xi, \cdot)\right)_{j=1,2}$ and $\left(\tilde{q}_{j}(\xi, \cdot)\right)_{j=1,2}$ of $L_{\xi}$ associated with $\lambda_{j}(\xi)$, of form, $j=1,2$,

$$
\begin{aligned}
& q_{j}(\xi, \cdot)=(i \bar{k} \xi)^{-1} \beta_{j, 1}(\xi) v_{1}(\xi, \cdot)+\beta_{j, 2}(\xi) v_{2}(\xi, \cdot) \\
& \tilde{q}_{j}(\xi, \cdot)=i \bar{k} \xi \quad \tilde{\beta}_{j, 1}(\xi) \tilde{v}_{1}(\xi, \cdot)+\tilde{\beta}_{j, 2}(\xi) \tilde{v}_{2}(\xi, \cdot)
\end{aligned}
$$

where

- $\left.j=1,2, v_{j}:\right]-\xi_{0}, \xi_{0}\left[\rightarrow L_{\mathrm{per}}^{2}([0,1])\right.$ and $\left.\tilde{v}_{j}:\right]-\xi_{0}, \xi_{0}\left[\rightarrow L_{\mathrm{per}}^{2}([0,1])\right.$ are analytic functions such that, when $|\xi|<\xi_{0},\left(v_{j}(\xi, \cdot)\right)_{j=1,2}$ and $\left(\tilde{v}_{j}(\xi, \cdot)\right)_{j=1,2}$ are dual bases of the total eigenspace of $L_{\xi}$ associated with spectrum $\sigma\left(L_{\xi}\right) \cap B\left(0, \varepsilon_{0}\right)$, and

$$
v_{1}(0, \cdot)=\bar{U}^{\prime}, \quad v_{2}(0, \cdot) \equiv 1 \quad \text { and } \quad \tilde{v}_{2}(0, \cdot) \equiv 1
$$

- $\left.j=1,2, k=1,2, \beta_{j, k}:\right]-\xi_{0}, \xi_{0}\left[\rightarrow \mathbb{C}\right.$ and $\left.\tilde{\beta}_{j, k}:\right]-\xi_{0}, \xi_{0}[\rightarrow \mathbb{C}$ are analytic and

$$
\beta_{j, 1}(0) \neq 0, \quad j=1,2 .
$$

Proof. Since 0 is separated from the rest of the spectrum of $L_{0}$, standard spectral theory for perturbations by relatively compact operators (see [14]) provides $\xi_{0}, \varepsilon_{0}$ and continuous $\lambda_{1}, \lambda_{2}$ such that, for $|\xi|<\xi_{0}, \sigma\left(L_{\xi}\right) \cap B\left(0, \varepsilon_{0}\right)=\left\{\lambda_{1}(\xi), \lambda_{2}(\xi)\right\}$.

Moreover this also yields analytic dual right and left spectral projectors associated to spectrum in $B\left(0, \varepsilon_{0}\right)$. Analytic dual bases of the right and left eigenspaces may then be obtained by appropriately continuing dual bases for spectral spaces of the spectrum of $L_{0}$ in $B\left(0, \varepsilon_{0}\right)$. We may choose such bases in the form $\left(\bar{U}^{\prime}, 1\right)$ and $(*, 1)$ and obtain in this way the $\left(v_{j}\right)$ and $\left(\tilde{v}_{j}\right)$ of the lemma.

We are thus left with the spectral analysis of

$$
M_{\xi}=\left[\left\langle\tilde{v}_{j}(\xi, \cdot), L_{\xi} v_{k}(\xi, \cdot)\right\rangle_{L^{2}([0,1]}\right]_{j, k},
$$

a $2 \times 2$ matrix perturbation problem. Still $M_{0}=\left(\begin{array}{cc}0 & -6 \bar{k} \\ 0 & 0\end{array}\right)$ but we will scale $M_{\xi}$ to blow up the double eigenvalue.

To do so, let us expand

$$
L_{\xi}=L_{0}+i \bar{k} \xi L^{(1)}+(i \bar{k} \xi)^{2} L^{(2)}+(i \bar{k} \xi)^{3} L^{(3)}+(i \bar{k} \xi)^{4} L^{(4)} .
$$

Specifically

$$
L^{(1)}=-(6 \bar{U}-\bar{c})-3 \bar{k}^{2} \partial_{x}^{2}-\delta\left(2 \bar{k} \partial_{x}+4 \bar{k}^{3} \partial_{x}^{3}\right) .
$$

Then notice that $\left\langle\tilde{v}_{2}(0, \cdot), L^{(1)} v_{1}\left(0_{\dot{1}}\right)\right\rangle=0$. This may be seen either by direct inspection or differentiating profile equation (2.5) with respect to $k$ (see $(3.7)$ ). Therefore

$$
M_{\xi} \stackrel{0}{=}\left(\begin{array}{cc}
0 & -6 \bar{k} \\
0 & 0
\end{array}\right)+i \bar{k} \xi\left(\begin{array}{cc}
* & * \\
0 & *
\end{array}\right)+\mathcal{O}\left(|\xi|^{2}\right) \text {. }
$$


As a consequence, the following scaling

rescale

$$
\check{M}_{\xi}:=(i \bar{k} \xi)^{-1} S(\xi) M_{\xi} S(\xi)^{-1}, \quad S(\xi):=\left(\begin{array}{cc}
i \bar{k} \xi & 0 \\
0 & 1
\end{array}\right)
$$

preserves smoothness in $\xi$. Since the eigenvalues $m_{j}(\xi)$ of $\check{M}_{\xi}$ are $(i \bar{k} \xi)^{-1} \lambda_{j}(\xi)$, their continuity provides the missing differentiability at 0 . As $m_{j}(0)=\lambda_{0, j}$, under assumption (B) eigenmodes of $\check{M}_{\xi}$ are analytic in $\xi$ in a neighborhood of 0 . Undoing the scaling finishes the proof except for the non vanishing condition on $\beta_{j, 1}(0), j=1,2$.

The only thing left is thus to explain why a right eigenvector of $\check{M}_{0}$ can not have 0 as first component. This follows from the structure $\check{M}_{0}=\left(\begin{array}{cc}* & -6 \bar{k} \\ * & *\end{array}\right)$.

\section{Expansion of eigenvalues}

Our next step is to obtain information about expansion of critical eigenmodes in a form suitable for comparison with modulation averaged equations. We assume (A') and (B).

In terms of the previous lemma, for $j=1,2$, we set

$$
\check{u}_{j}(\xi, \cdot)=i \bar{k} \xi q(\xi, \cdot), \quad k_{0, j}=\beta_{j, 1}(0),
$$

and are looking for terms in expansions

$$
\begin{aligned}
\lambda_{j}(\xi) & =i \bar{k} \xi \lambda_{0, j}+(i \bar{k} \xi)^{2} \lambda_{1, j}+(i \bar{k} \xi)^{3} \lambda_{2, j}+\mathcal{O}\left(\xi^{4}\right) \\
\check{u}_{j}(\xi, \cdot) & =k_{0, j} \bar{U}^{\prime}+(i \bar{k} \xi) \check{u}_{1, j}(\xi, \cdot)+(i \bar{k} \xi)^{2} \check{u}_{2, j}(\xi, \cdot)+(i \bar{k} \xi)^{3} \check{u}_{3, j}(\xi, \cdot)+\mathcal{O}\left(\xi^{4}\right)
\end{aligned}
$$

by inspection of

$$
L_{\xi} \check{u}_{j}(\xi, \cdot)=\lambda_{j}(\xi) \check{u}_{j}(\xi, \cdot) .
$$

For the sake of simplicity we drop the $j$-dependence in the following computations.

Recall notations of $\left(\frac{\text { Lert }}{3.2)}\right.$. First nontrivial step is

$$
L_{0}\left[\check{u}_{1}\right]=-k_{0} L^{(1)} \bar{U}^{\prime}+k_{0} \lambda_{0} \bar{U}^{\prime} .
$$

To simplify the equation we use

$$
L_{0} \partial_{k} \bar{U}+\bar{k} \partial_{k} \bar{c} \bar{U}^{\prime}+L^{(1)} \bar{U}^{\prime}=0,
$$

obtained differentiating profile equation $(2.5)$ with respect to $k$, and the already known

$\mathrm{dM}$

$$
L_{0} \partial_{M} \bar{U}+\bar{k} \partial_{M} \bar{c} \bar{U}^{\prime}=0
$$

obtained differentiating profile equation (2.5II with respect to $M$. This yields for any choice of $M_{0}$

$$
L_{0}\left(\check{u}_{1}-\mathrm{d} \bar{U}\left[k_{0}, M_{0}\right]\right)+\left(\lambda_{0} k_{0}+\bar{k} \mathrm{~d} \bar{c}\left[k_{0}, M_{0}\right]\right) \bar{U}^{\prime}=0
$$


(where $\mathrm{d}$ applied to profiles, phase velocity and time frequency stands for the total derivative with respect to parameters $(k, M)$ and $\cdot$ denotes as before evaluation in $(\bar{k}, \bar{M}))$. We choose $M_{0}$ so that

cond_10 (3.10)

$$
\lambda_{0} k_{0}+\bar{k} \mathrm{~d} \bar{c}\left[k_{0}, M_{0}\right]=0 .
$$

This is possible and determines $M_{0}$ since $\partial_{M} \bar{c}=6 \neq 0$.

To go beyond this level of description, we need to make some normalizing choices. To prevent confusion, we temporarily mark again the $j$-dependence. For $j=1,2$, since $k_{0, j} \neq 0$, we may impose, up to changing $\xi_{0}$ again, for $|\xi|<\xi_{0}$,

norm_eig

$$
\left\langle\tilde{v}_{1}(0, \cdot), \check{u}_{j}(\xi, \cdot)\right\rangle=k_{0, j} .
$$

Moreover up to changing parameterization by a $k$-dependent shift, we may also impose

(N) $\left\langle\tilde{v}_{1}(0, \cdot), \partial_{k} \bar{U}\right\rangle=0$.

With these choices, since also holds $\left\langle\tilde{v}_{1}(0, \cdot), \partial_{M} \bar{U}\right\rangle=\left\langle\tilde{v}_{1}(0, \cdot), v_{2}(0, \cdot)\right\rangle=0$, equations $(3.9)$ and (3.10) imply

bar_u1 (3.12)

$$
\check{u}_{1}=\mathrm{d} \bar{U}\left[k_{0}, M_{0}\right] .
$$

Incidentally notice that indeed $M_{0}=\left\langle\check{u}_{1}\right\rangle$.

Next step in expansion of $(3.5)$ is

nu2

$$
L_{0}\left[\check{u}_{2}\right]=-L^{(1)}\left(\mathrm{d} \bar{U}\left[k_{0}, M_{0}\right]\right)-k_{0} L^{(2)} \bar{U}^{\prime}+\lambda_{0} \mathrm{~d} \bar{U}\left[k_{0}, M_{0}\right]+k_{0} \lambda_{1} \bar{U}^{\prime} .
$$

Solvability condition is found taking the scalar product with $\tilde{v}_{2}(0, \cdot) \equiv 1$ and reduces to

$$
\lambda_{0} M_{0}+\mathrm{d}\left[\overline{\left\langle 3 U^{2}\right\rangle}\right]\left[k_{0}, M_{0}\right]-\bar{c} M_{0}=0
$$

(recall $(\stackrel{\mathrm{Ls}}{3.3}))$. Mark that $\left(\right.$ cond_dond 20 (3.10,3.14) forms an eigenvalue problem in $\lambda_{0}$ and $\left(k_{0}, M_{0}\right)$,

displ_0

$$
\lambda_{0}\left(\begin{array}{c}
k_{0} \\
M_{0}
\end{array}\right)=\left(\begin{array}{cc}
-\bar{k} \partial_{k} \bar{c} & -\bar{k} \partial_{M} \bar{c} \\
-\partial_{k}\left[\overline{\left\langle 3 U^{2}\right\rangle}\right] & -\partial_{M}\left[\overline{\left\langle 3 U^{2}\right\rangle}\right]+\bar{c}
\end{array}\right)\left(\begin{array}{c}
k_{0} \\
M_{0}
\end{array}\right)
$$

Notice that, since $-\bar{k} \partial_{M} \bar{c} \neq 0$, once $\left(\lambda_{0}, k_{0}\right)$ is fixed, $M_{0}$ is determined by the previous relation.

Since solvability conditions are met, we may introduce, for any $\lambda \in \mathbb{C}, \check{f}^{k}(\lambda ; \cdot)$ and $\check{f}^{M}(\lambda ; \cdot)$ the solutions of

checkf_k

checkf_M

$$
\begin{aligned}
L_{0}\left(\check{f}^{k}(\lambda ; \cdot)\right) & =-L^{(1)}\left(\partial_{k} \bar{U}\right)-\partial_{k}\left[\overline{\left\langle 3 U^{2}\right\rangle}\right]-L^{(2)} \bar{U}^{\prime}+\lambda \partial_{k} \bar{U} \\
L_{0}\left(\check{f}^{M}(\lambda ; \cdot)\right) & =-L^{(1)}\left(\partial_{M} \bar{U}\right)-\partial_{M}\left[\overline{\left\langle 3 U^{2}\right\rangle}\right]+\bar{c}+\lambda \partial_{M} \bar{U}-\lambda
\end{aligned}
$$

orthogonal to $\tilde{v}_{1}(0, \cdot)$. In view of $\left(\frac{\text { cond } 20}{3.14)}\right.$ and $\left(\frac{\mathrm{dM}}{3.8}\right)$, this enables us to rewrite $\left(\frac{\mathrm{nu} 2}{3.13}\right)$ in the compact form

$$
L_{0}\left(\check{u}_{2}-\partial_{M} \bar{U} \tilde{M}_{1}-\check{f}\left(\lambda_{0}\right)\left[k_{0}, M_{0}\right]\right)-\left(\lambda_{1} k_{0}+\bar{k} \partial_{M} \bar{c} \tilde{M}_{1}\right) \bar{U}^{\prime}=0
$$


for any choice of $\tilde{M}_{1}$. Choosing $\tilde{M}_{1}$ to solve

lde_cond_11

bar_u2

ilde_M1_eig

cond_11

nu3

cond_21

$$
\lambda_{1} k_{0}+\bar{k} \partial_{M} \bar{c} \tilde{M}_{1}=0,
$$

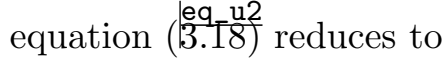

$$
\check{u}_{2}=\partial_{M} \bar{U} \tilde{M}_{1}+\check{f}\left(\lambda_{0}\right)\left[k_{0}, M_{0}\right] .
$$

thanks to normalization ( $\left(\text { norm }^{\text {eig }}\right)_{\text {. }}$. Notice that the absence of a $k_{1}$ parameter is also due to our normalization choices and that a priori $\tilde{M}_{1}$ is not $\left\langle\check{u}_{2}\right\rangle$. Let us set $M_{1}=\left\langle\check{u}_{2}\right\rangle$. Then

$$
M_{1}=\tilde{M}_{1}+\left\langle\check{f}\left(\lambda_{0}\right)\right\rangle\left[k_{0}, M_{0}\right]
$$

and

$$
\lambda_{1} k_{0}+\bar{k} \partial_{M} \bar{c} M_{1}=\bar{k} \partial_{M} \bar{c}\left\langle\check{f}\left(\lambda_{0}\right)\right\rangle\left[k_{0}, M_{0}\right] .
$$

In the expansion of $\left(\frac{g K S}{3.5}\right)$, we now come to

$$
L_{0}\left[\check{u}_{3}\right]=-L^{(1)} \check{u}_{2}-L^{(2)}\left(\mathrm{d} \bar{U}\left[k_{0}, M_{0}\right]\right)-k_{0} L^{(3)} \bar{U}^{\prime}+\lambda_{0} \check{u}_{2}+\lambda_{1} \mathrm{~d} \bar{U}\left[k_{0}, M_{0}\right]+k_{0} \lambda_{2} \bar{U}^{\prime}
$$

Solvability condition for this equation writes

$$
\begin{aligned}
\lambda_{1} M_{0} & +\lambda_{0} M_{1}+\left(\partial_{M}\left[\overline{\left\langle 3 U^{2}\right\rangle}\right]-\bar{c}\right) M_{1}-\left(\partial_{M}\left[\overline{\left\langle 3 U^{2}\right\rangle}\right]-\bar{c}\right)\left\langle\check{f}\left(\lambda_{0}\right)\right\rangle\left[k_{0}, M_{0}\right] \\
& =-\left\langle(6 \bar{U}-\bar{c}) \check{f}\left(\lambda_{0}\right)\right\rangle\left[k_{0}, M_{0}\right]-\delta M_{0} .
\end{aligned}
$$

We stop here the analysis of the expansions and discuss now to which extent the obtained relations determine the parameters appearing in the expansions. $\lambda_{0,1}$ and $\lambda_{0,2}$ are the eigenvalues of the matrix appearing in (3.15). Pick one $j \in 1,2$ and drop the $j$-dependence. We have left some arbitrariness in the choice of $k_{0}$ but we can normalize it to 1 . Then $M_{0}$ is uniquely determined. We still have to explain why this is also true for $M_{1}$ and $\lambda_{1}$. Since their coefficients in (3.22) are both nonzero, in view of (3.24) this amounts to prove that we

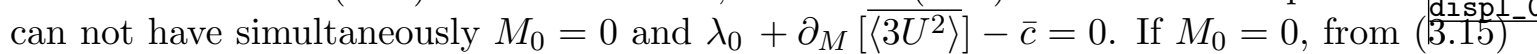
we deduce $\lambda_{0}=-\bar{k} \partial_{k} \bar{c}$ and $\partial_{k}\left[\overline{\left\langle 3 U^{2}\right\rangle}\right]=0$ thus $\left\{\lambda_{0,1}, \lambda_{0,2}\right\}=\left\{-\bar{k} \partial_{k} \bar{c},-\partial_{M}\left[\overline{\left\langle 3 U^{2}\right\rangle}\right]+\bar{c}\right\}$ and

$$
\lambda_{0}=-\bar{k} \partial_{k} \bar{c} \neq-\partial_{M}\left[\overline{\left\langle 3 U^{2}\right\rangle}\right]+\bar{c} .
$$

Therefore $k_{1}$ and $M_{1}$ are uniquely determined.

The process described here may be carried out by induction up to any order. We have chosen to stop at the first order that may be nontrivial and stable, that is at an order at which the expansions of $\breve{u}_{1}(\xi, \cdot)$ and $\breve{u}_{2}(\xi, \cdot)$ are not collinear and the expansions of eigenvalues may have a negative real part (both for nonzero Floquet). 


\section{Modulation systems}

We now derive formally the modulation averaged equations to which we will compare the critical low-Floquet expansions.

Although we call Whitham's systems the obtained equations, we will not develop the approach proposed in $[25]$ since it is designed for Lagrangian systems. Instead we follow a nonlinear WKB approach close to the one adopted in [21] by Serre. Let us stress that both [25] and [21] are only concerned with the formal derivation of a first-order modulation system.

\section{First order}

We make the derivation as general as possible and therefore a priori not restricted to perturbation of a given wave, so that we are back to the original frame, instead of a co-moving one. We are looking for a formal expansion of a solution $u$ of equation (1.1) according to the two-scale ansatz

$$
u(x, t)=U\left(\frac{\phi(\varepsilon x, \varepsilon t)}{\varepsilon} ; \varepsilon x, \varepsilon t\right)
$$

where

ansWKB

$$
U(y, X, T)=\sum_{j} \varepsilon^{j} U_{j}(y ; X, T) \quad \text { and } \quad \phi(X, T)=\sum_{j} \varepsilon^{j} \phi_{j}(X, T)
$$

with all functions of period 1 in the $y$-variable. We insert the ansatz $(3.25,3.26)$ into (II.I $)$ and collect terms of the same order in $\varepsilon$.

At first this yields, with $\Omega_{0}=\partial_{T} \phi_{0}$ and $k_{0}=\partial_{X} \phi_{0}$,

$$
\Omega_{0} \partial_{y} U_{0}+k_{0} \partial_{y}\left(3 U_{0}^{2}\right)+k_{0}^{3} \partial_{y}^{3} U_{0}+\delta\left(k_{0}^{2} \partial_{y}^{2} U_{0}+k_{0}^{4} \partial_{y}^{4} U_{0}\right)=0
$$

Assuming $k_{0}$ and $M_{0}=\int_{0}^{1} U_{0}(y ; \cdot, \cdot)$ dy are valued in the open set covered by assumption (A') we solve $(\overline{3.27})$ with

solve_me_0

$$
\begin{aligned}
\Omega_{0}(X, T) & =-k_{0}(X, T) c\left(k_{0}(X, T), M_{0}(X, T)\right) \\
U_{0}(y ; X, T) & =U\left(y, k_{0}(X, T), M_{0}(X, T)\right) .
\end{aligned}
$$

The compatibility condition $\partial_{T} \partial_{X} \phi_{0}=\partial_{X} \partial_{T} \phi_{0}$ yields the first equation of a Whitham's modulation system

$$
\partial_{T} k_{0}+\partial_{X}\left(k_{0} c\left(k_{0}, M_{0}\right)\right)=0 .
$$

We have disregarded in (3.28) the possibility of a phase shift dependent on $(X, T)$ since $\phi_{1}$ already encodes this possibility. We will have to rule out similar problems of uniqueness in the following steps. 


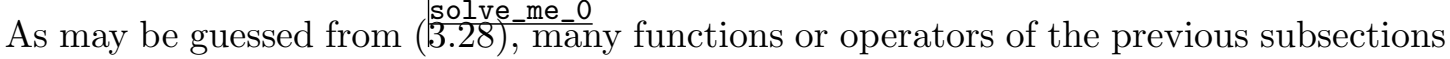
that were there evaluated at $(\bar{k}, \bar{M})$ will appear here evaluated at $\left(k_{0}(X, T), M_{0}(X, T)\right)$, and for operators they act here on the variable $y$ only. We will use for them the notations of the previous subsections without explicit dependence on $\left(k_{0}(X, T), M_{0}(X, T)\right)$ when no confusion is possible and with a subscript $\cdot_{0}$ when needed. Requiring that $k_{0}$ and $M_{0}$ are valued in the open set covered by both assumptions (A') and (B), this dependence in parameters is smooth. With this settled, we fix some of the uniqueness issues of the ansatz by adding the condition

norm_ans

$$
\left\langle\tilde{v}_{1}(0, \cdot), U_{j}(\cdot ; X, T)\right\rangle=0, \quad j \neq 0,
$$

(recall lemma 3.1).

$$
\text { blochfacts }
$$

Next step of the identification process gives, with $\Omega_{1}=\partial_{T} \phi_{1}$ and $k_{1}=\partial_{X} \phi_{1}$,

$$
\begin{aligned}
\left(\Omega_{1}+c_{0} k_{1}\right) U_{0}^{\prime} & -k_{1} L^{(1)} U_{0}^{\prime}-L_{0} U_{1}-L^{(1)} \partial_{X} U_{0} \\
& -\partial_{X} k_{0} L^{(2)} U_{0}^{\prime}+\partial_{T} U_{0}+c_{0} \partial_{X} U_{0}=0,
\end{aligned}
$$

where' denotes $\partial_{y}$. Solvability condition for $L_{0}$ reads

$$
\partial_{T} M_{0}+\partial_{X}\left(\left\langle 3 U^{2}\left(. ; k_{0}, M_{0}\right)\right\rangle\right)=0 .
$$

For concision's sake we denote the averaged flux

$$
F(k, M)=\left\langle 3 U^{2}\left(. ; k_{0}, M_{0}\right)\right\rangle .
$$

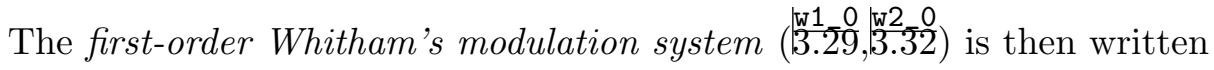

w12_0

$$
\left\{\begin{array}{c}
\partial_{T} k_{0}+\partial_{X}\left(k_{0} c\left(k_{0}, M_{0}\right)\right)=0 \\
\partial_{T} M_{0}+\partial_{X}\left(F\left(k_{0}, M_{0}\right)\right)=0
\end{array}\right.
$$

Before going on with the derivation process, we write down the eigenvalue problem corresponding to ( $(\overline{3.34})$. Linearizing ( $(\overline{3.34})$ about $(\bar{k}, \bar{M})$ in the frame $(\bar{k}(X-\bar{c} T), T)$ yields

wlin_0

$$
\left\{\begin{array}{cc}
\partial_{T} k+\bar{k} \partial_{X}(\bar{k} \mathrm{~d} \bar{c}(k, M)) & =0 \\
\partial_{T} M+\bar{k} \partial_{X}(\mathrm{~d} \bar{F}(k, M)-\bar{c} M) & =0
\end{array}\right.
$$

Hence, applying Fourier transform, the eigenvalue problem

dispw_0

$$
\lambda(\xi)\left(\begin{array}{c}
\hat{k}(\xi) \\
\hat{M}(\xi)
\end{array}\right)=i \bar{k} \xi\left(\begin{array}{cc}
-\bar{k} \partial_{k} \bar{c} & -\bar{k} \partial_{M} \bar{c} \\
-\partial_{k} \bar{F} & -\partial_{M} \bar{F}+\bar{c}
\end{array}\right)\left(\begin{array}{c}
\hat{k}(\xi) \\
\hat{M}(\xi)
\end{array}\right) .
$$




\section{Second order}

To proceed, for arbitrary $(\bar{k}, \bar{M})$ we introduce $g^{k}(\bar{k}, \bar{M} ; \cdot), g^{M}(k, M ; \cdot)$ the solutions of

g_k

g_M

$m e+1$

Om1

solve_me_1

$$
\begin{aligned}
L_{0}\left(g^{k}(\bar{k}, \bar{M} ; \cdot)\right)= & -L^{(1)} \partial_{k} \bar{U}-\partial_{k} \bar{F}-L^{(2)} \bar{U}^{\prime} \\
& -\partial_{k} \bar{U} \bar{k} \partial_{k} \bar{c}-\left(\partial_{M} \bar{U}-1\right) \partial_{k} \bar{F} \\
L_{0}\left(g^{M}(\bar{k}, \bar{M} ; \cdot)\right)= & -L^{(1)} \partial_{M} U_{0}-\partial_{M} \bar{F}+\bar{c} \\
& -\partial_{k} \bar{U} \bar{k} \partial_{M} \bar{c}-\left(\partial_{M} \bar{U}-1\right)\left[\partial_{M} \bar{F}-\bar{c}\right]
\end{aligned}
$$

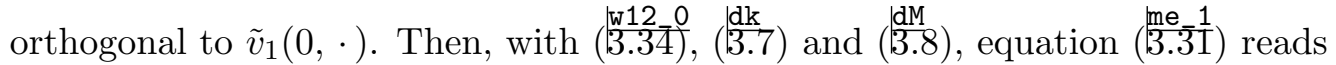

$$
L_{0}\left(U_{1}-\mathrm{d} U_{0}\left(k_{1}, \tilde{M}_{1}\right)-g_{0}\left[\partial_{X} k_{0}, \partial_{X} M_{0}\right]\right)=\left(\Omega_{1}+k_{0} \mathrm{~d} c_{0}\left[k_{1}, \tilde{M}_{1}\right]+c_{0} k_{1}\right) U_{0}^{\prime}
$$

for any choice of $\tilde{M}_{1}$. Choosing $\tilde{M}_{1}$ to get

$$
\Omega_{1}+k_{0} \mathrm{~d} c_{0}\left[k_{1}, \tilde{M}_{1}\right]+c_{0} k_{1}=0
$$

and normalizing parameterization according to $(\mathrm{N}),\left(\frac{\mathrm{me}}{3.39}\right)$ is reduced to

$$
U_{1}=\mathrm{d} U_{0}\left(k_{1}, \tilde{M}_{1}\right)+g_{0}\left[\partial_{X} k_{0}, \partial_{X} M_{0}\right] .
$$

Let us set $M_{1}=\left\langle U_{1}\right\rangle$. Then

$$
M_{1}=\tilde{M}_{1}+\left\langle g_{0}\right\rangle\left[\partial_{X} k_{0}, \partial_{X} M_{0}\right]
$$

and compatibility condition $\partial_{T} k_{1}=\partial_{X} \Omega_{1}$ yields

$$
\partial_{T} k_{1}+\partial_{X}\left(k_{0} \mathrm{~d} c_{0}\left[k_{1}, M_{1}\right]+c_{0} k_{1}\right)=\partial_{X}\left(k_{0} \partial_{M} c_{0}\left\langle g_{0}\right\rangle\left[\partial_{X} k_{0}, \partial_{X} M_{0}\right]\right) .
$$

Back to the identification process, we obtain

$$
\begin{aligned}
\left(\Omega_{2}+c_{0} k_{2}\right) U_{0}^{\prime} & -k_{2} L^{(1)} U_{0}^{\prime}-L_{0} U_{1}-L^{(1)} \partial_{X} U_{1} \\
& -k_{1}^{2} L^{(2)} U_{0}^{\prime \prime}-k_{1} L^{(1)} U_{1}^{\prime}-L^{(2)} \partial_{X}^{2} U_{0} \\
& -\partial_{X} k_{0} L^{(2)} U_{1}^{\prime}-\partial_{X} k_{1} L^{(2)} U_{0}^{\prime}-2 k_{1} L^{(2)} \partial_{X} U_{0}^{\prime} \\
& -\partial_{X}^{2} k_{0} L^{(3)} U_{0}^{\prime}-\partial_{X} k_{0} L^{(3)} \partial_{X} U_{0}^{\prime}-3\left(\partial_{X} k_{0}\right)^{2} L^{(4)} U_{0}^{\prime \prime} \\
& +\left(\Omega_{1}+c_{0} k_{1}\right) U_{1}^{\prime}+\partial_{T} U_{1}+c_{0} \partial_{X} U_{1} \\
& +6 U_{1} \partial_{X} U_{0}+3 k_{0}\left(U_{1}^{2}\right)^{\prime}+6 k_{1} U_{1} U_{0}^{\prime} \\
& +6 \delta k_{1} \partial_{X}\left(k_{0}^{2}\right) U_{0}^{\prime \prime \prime}+2 k_{1} \partial_{X} k_{0} U_{0}^{\prime \prime \prime}=0
\end{aligned}
$$


an equation of the form

$$
\partial_{T} U_{1}+\partial_{X}\left(6 U_{0} U_{1}\right)+\delta \partial_{X}^{2} U_{0}+\partial_{y}(\cdots)=0
$$

whose solvability condition is

$$
\begin{aligned}
\partial_{T} M_{1}+\partial_{X}\left(\mathrm{~d} F_{0}\left[k_{1}, M_{1}\right]\right)= & -\delta \partial_{X}^{2} M_{0}-\partial_{X}\left(\left\langle 6 U_{0} g_{0}\right\rangle\left[\partial_{X} k_{0}, \partial_{X} M_{0}\right]\right) \\
& +\partial_{X}\left(\partial_{M} F_{0}\left\langle g_{0}\right\rangle\left[\partial_{X} k_{0}, \partial_{X} M_{0}\right]\right) .
\end{aligned}
$$

To write the second order system in a compact form, let us introduce

diff_coeff

$$
\begin{aligned}
& d_{1,1}(k, M)=k \partial_{M} c(k, M)\left\langle g^{k}(k, M)\right\rangle \\
& d_{1,2}(k, M)=k \partial_{M} c(k, M)\left\langle g^{M}(k, M)\right\rangle \\
& d_{2,1}(k, M)=-\left\langle 6 U(k, M) g^{k}(k, M)\right\rangle+\partial_{M} F(k, M)\left\langle g^{k}(k, M)\right\rangle \\
& d_{2,2}(k, M)=-\delta-\left\langle 6 U(k, M) g^{M}(k, M)\right\rangle+\partial_{M} F(k, M)\left\langle g^{M}(k, M)\right\rangle
\end{aligned}
$$

Remark that equations $\left(\frac{W_{1}-0}{3.29}, \frac{W 2}{3.32}, \frac{W 1}{3} \frac{1}{3}, \frac{1}{3} 2 \frac{1}{3.45}\right)$ are the first equations obtained in the formal expansion of a solution $(\kappa, \mathcal{M})$ of

w12_1

$$
\left\{\begin{aligned}
\partial_{t} \kappa+\partial_{x}(\kappa c(\kappa, \mathcal{M})) & =\partial_{x}\left(d_{1,1}(\kappa, \mathcal{M}) \partial_{x} \kappa+d_{1,2}(\kappa, \mathcal{M}) \partial_{x} \mathcal{M}\right) \\
\partial_{t} \mathcal{M}+\partial_{x}(F(\kappa, \mathcal{M})) & =\partial_{x}\left(d_{2,1}(\kappa, \mathcal{M}) \partial_{x} \kappa+d_{2,2}(\kappa, \mathcal{M}) \partial_{x} \mathcal{M}\right)
\end{aligned}\right.
$$

according to the low-frequency ansatz

$$
(\kappa, \mathcal{M})(x, t)=(k, M)(\varepsilon x, \varepsilon t) \quad \text { where } \quad(k, M)(X, T)=\sum_{j} \varepsilon^{j}\left(k_{j}, M_{j}\right)(X, T) .
$$

We call system (3.47) a second-order Whitham's modulation system. Note that, in contrast with the present situation, going back to physical variables $(x, t)$ would have been of no effect on the shape of system (3.34), hence we have skipped the unscaling step there. Now we write down the eigenvalue problem corresponding to $(3.47)$. Linearizing $(3.47)$ about $(\bar{k}, \bar{M})$ in the frame $(\bar{k}(x-\bar{c} t), t)$ yields

wlin_1

$$
\left\{\begin{aligned}
\partial_{t} k+\bar{k} \partial_{x}(\bar{k} \mathrm{~d} \bar{c}(k, M)) & =\bar{k} \partial_{x}\left(\bar{d}_{1,1} \bar{k} \partial_{x} k+\bar{d}_{1,2} \bar{k} \partial_{x} M\right) \\
\partial_{t} M+\bar{k} \partial_{x}(\mathrm{~d} \bar{F}(k, M)-\bar{c} M) & =\bar{k} \partial_{x}\left(\bar{d}_{2,1} \bar{k} \partial_{x} k+\bar{d}_{2,2} \bar{k} \partial_{x} M\right)
\end{aligned}\right.
$$

Hence, applying Fourier transform, the eigenvalue problem

dispw_1

$$
\lambda(\xi)\left(\begin{array}{c}
\hat{k}(\xi) \\
\hat{M}(\xi)
\end{array}\right)=\left[i \bar{k} \xi\left(\begin{array}{cc}
-\bar{k} \partial_{k} \bar{c} & -\bar{k} \partial_{M} \bar{c} \\
-\partial_{k} \bar{F} & -\partial_{M} \bar{F}+\bar{c}
\end{array}\right)+(i \bar{k} \xi)^{2}\left(\begin{array}{cc}
\bar{d}_{1,1} & \bar{d}_{1,2} \\
\bar{d}_{1,1} & \bar{d}_{1,2}
\end{array}\right)\right]\left(\begin{array}{c}
\hat{k}(\xi) \\
\hat{M}(\xi)
\end{array}\right)
$$




\section{Statements}

Now we look back at expansions of critical eigenmodes to validate at the spectral level the derived modulation systems.

main Theorem 3.2. Assume $\left(A^{\prime}\right)$ and $(B)$ and adopt notations of lemma ${ }^{\text {blochfacts }}$

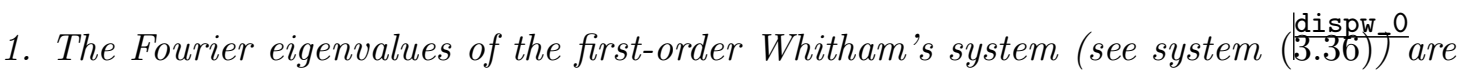
$i \bar{k} \xi \lambda_{0,1}$ and $i \bar{k} \xi \lambda_{0,2}$. Moreover choosing corresponding eigenvectors $\left(k_{0}^{(1)}, M_{0}^{(1)}\right)$ and $\left(k_{0}^{(2)}, M_{0}^{(2)}\right)$, and normalizing parameterization according to $(N)$, we may normalize right eigenfunctions in such a way that, for $j=1,2$,

eigf_expand_0

$$
q_{j}(\xi, \cdot) \stackrel{0}{=} \frac{1}{i \bar{k} \xi} k_{0}^{(j)} \bar{U}^{\prime}+\mathrm{d} \bar{U}\left(k_{0}^{(j)}, M_{0}^{(j)}\right)+\mathcal{O}(\xi)
$$

2. Normalize parameterization according to $(N)$. In a neighborhood of the origin, the Fourier modes of the second-order Whitham's system (see system (3.50) T are analytic functions of the Fourier frequency. For small Fourier frequency $\xi$, we may label these eigenvalues as $i \bar{k} \xi \mu_{1}(\xi)$ and $i \bar{k} \xi \mu_{2}(\xi)$ in such a way that, for $j=1,2$,

eigv_expand_1

$$
\lambda_{j}(\xi) \stackrel{0}{=} i \bar{k} \xi \mu_{j}(\xi)+\mathcal{O}\left(\xi^{3}\right) .
$$

and normalize corresponding eigenmodes $\left(k^{(1)}(\xi), M^{(1)}(\xi)\right)$ and $\left(k^{(2)}(\xi), M^{(2)}(\xi)\right)$ and eigenfunctions in such a way that, for $j=1,2$,

$$
\begin{aligned}
\left(\begin{array}{c}
k^{(j)}(\xi) \\
M^{(j)}(\xi)
\end{array}\right) & \stackrel{0}{=}\left(\begin{array}{c}
k_{0}^{(j)} \\
M_{0}^{(j)}
\end{array}\right)+i \bar{k} \xi\left(\begin{array}{c}
0 \\
M_{1}^{(j)}
\end{array}\right)+\mathcal{O}\left(\xi^{2}\right) \\
q_{j}(\xi, \cdot) & \stackrel{0}{=} \frac{1}{i \bar{k} \xi} k^{(j)_{0}} \bar{U}^{\prime}+\mathrm{d} \overline{\mathrm{U}}\left(\mathrm{k}^{(\mathrm{j})_{0}}, \mathrm{M}_{0}^{(\mathrm{j})}\right) \\
& +i \bar{k} \xi \partial_{M} \bar{U}\left(M_{1}^{(j)}-\langle\bar{g}\rangle\left[k_{0}^{(j)}, M_{0}^{(j)}\right]\right) \\
& +i \bar{k} \xi \bar{g}\left[k_{0}^{(j)}, M_{0}^{(j)}\right]+\mathcal{O}\left(\xi^{2}\right)
\end{aligned}
$$

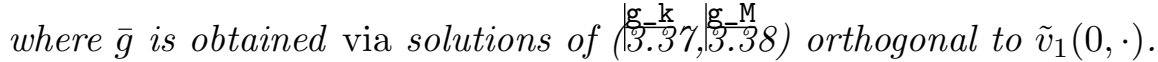

Proof. We first remark that the singularity at $\xi=0$ in $\left(\frac{d i s p w-0}{3.36)}\right.$ and $\frac{\text { dispw }-1}{3.50}=1$ artificial, as may be seen by diyiding by $i \bar{k} \xi$ and setting $\mu=\lambda /(i \bar{k} \xi)$. In this way, (3.36) 1 is made independent of $\xi$ and in (3.50) we are looking for $\mu_{j}(\xi)$.

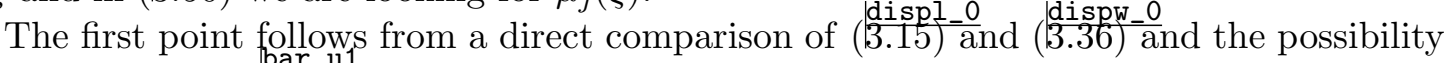
to prescribe $k_{\text {din }_{\text {in }}\left(\mathrm{B}_{1} .12\right) \text {. }}$

Problem $(3.50)$ is a low-frequency perturbation of $\left(\frac{d i s p w-0}{3.36}\right)$. To prove the second point we fix $j$ and expand $\mu_{j}(\xi)$ and $\left(k^{(j)}(\xi), M^{(j)}(\xi)\right)$ according to the fact that $\left(i \bar{k} \xi \mu_{j}(\xi), k^{(j)}(\xi), M^{(j)}(\xi)\right)$ solves (3.50) and $\mu_{j}(0)=\lambda_{0, j}$. Since $\mu_{1}(0) \neq \mu_{2}(0)$, expansions are regular in a neighborhood of 0 . As necessarily $k^{(j)}(0)$ is nonzero, we may normalize eigenvectors to impose 
$k^{(j)}(\xi)=k_{0}^{(j)}$ thus

$$
\begin{aligned}
& \mu_{j}(0) \stackrel{0}{=} \lambda_{0, j}+i \bar{k} \xi \mu_{1, j}+\mathcal{O}\left(\xi^{2}\right) \\
&\left(\begin{array}{c}
k^{(j)}(\xi) \\
M^{(j)}(\xi)
\end{array}\right) \stackrel{0}{=}\left(\begin{array}{c}
k_{0}^{(j)} \\
M_{0}^{(j)}
\end{array}\right)+i \bar{k} \xi\left(\begin{array}{c}
0 \\
M_{1}^{(j)}
\end{array}\right)+\mathcal{O}\left(\xi^{2}\right) .
\end{aligned}
$$

This leads to

$\mathrm{mu} \_1$

$$
\begin{aligned}
\lambda_{0, j}\left(\begin{array}{c}
0 \\
M_{1}^{(j)}
\end{array}\right) & +\left[\begin{array}{cc}
\bar{k} \partial_{k} \bar{c} & \bar{k} \partial_{M} \bar{c} \\
\partial_{k} \bar{F} & \partial_{M} \bar{F}-\bar{c}
\end{array}\right]\left(\begin{array}{c}
0 \\
M_{1}^{(j)}
\end{array}\right)+\mu_{1, j}\left(\begin{array}{c}
k_{0}^{(j)} \\
M_{0}^{(j)}
\end{array}\right) \\
& =\left[\begin{array}{cc}
\bar{d}_{1,1} & \bar{d}_{1,2} \\
\bar{d}_{2,1} & \bar{d}_{2,2}
\end{array}\right]\left(\begin{array}{c}
k_{0}^{(j)} \\
M_{0}^{(j)}
\end{array}\right) .
\end{aligned}
$$

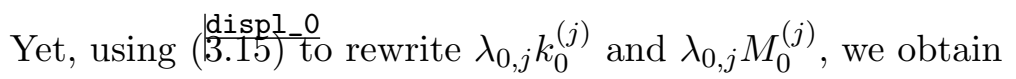

$$
\check{f}\left(\lambda_{0, j}\right)\left[k_{0}^{(j)}, M_{0}^{(j)}\right]=g(\bar{k}, \bar{M})\left[k_{0}^{(j)}, M_{0}^{(j)}\right]
$$

yielding

$$
\begin{aligned}
\bar{d}_{1,1} k_{0}^{(j)}+\bar{d}_{1,2} M_{0}^{(j)} & =\bar{k} \partial_{M} \bar{c}\left\langle\check{f}\left(\lambda_{0, j}\right)\right\rangle\left[k_{0}^{(j)}, M_{0}^{(j)}\right], \\
\bar{d}_{2,1} k_{0}^{(j)}+\bar{d}_{2,2} M_{0}^{(j)} & =\left(\partial_{M} \bar{F}-\bar{c}\right)\left\langle\check{f}\left(\lambda_{0, j}\right)\right\rangle\left[k_{0}^{(j)}, M_{0}^{(j)}\right] \\
- & \left\langle(6 \bar{U}-\bar{c}) \check{f}\left(\lambda_{0, j}\right)\right\rangle\left[k_{0}^{(j)}, M_{0}^{(j)}\right]-\delta M_{0}^{(j)} .
\end{aligned}
$$

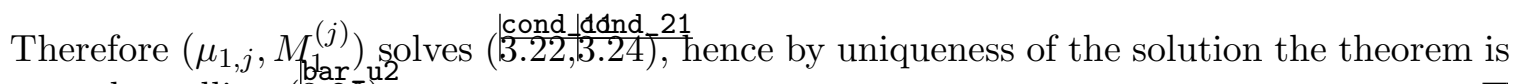
proved recalling $\left(\frac{\mathrm{bar}}{3.20)}\right.$.

Having lemma 3.1 in mind, one may ask whether something remains from theorem main if assumption (B) is removed. The answer is positive, assuming (A') only it may be proved that the characteristic speeds of the first-order Whitham's system are the $\lambda_{0, j}$ appearing in $(3.1)$.

submain Proposition 3.3. Assume $\left(A^{\prime}\right)$ and adopt notations of lemma $\frac{\text { blochfacts }}{3.1}$

The Fourier eigenvalues of the first-order Whitham's system (see system (dispw) ${ }^{0}$ are $i \bar{k} \xi \lambda_{0,1}$ and $i \bar{k} \xi \lambda_{0,2}$.

Proof. Coming back to the proof of lemma 3.1 , we know that $\lambda_{0,1}$ and $\lambda_{0,2}$ are the eigenvalues of

$$
\check{M}_{0}=\left(\begin{array}{cc}
\frac{1}{i \bar{k}}\left\langle\tilde{v}_{1}(0), L_{0} \partial_{\xi} v_{1}(0)\right\rangle+\left\langle\tilde{v}_{1}(0), L^{(1)} v_{1}(0)\right\rangle & \left\langle\tilde{v}_{1}(0), L_{0} v_{2}(0)\right\rangle \\
\frac{1}{(i \bar{k})^{2}}\left\langle\partial_{\xi} \tilde{v}_{2}(0), L_{0} \partial_{\xi} v_{1}(0)\right\rangle+\frac{1}{i k}\left\langle\tilde{v}_{2}(0), L^{(1)} \partial_{\xi} v_{1}(0)\right\rangle & \frac{1}{i \bar{k}}\left\langle\partial_{\xi} \tilde{v}_{2}(0), L_{0} v_{2}(0)\right\rangle \\
+\frac{1}{i \bar{k}}\left\langle\partial_{\xi} \tilde{v}_{2}(0), L^{(1)} v_{1}(0)\right\rangle+\left\langle\tilde{v}_{2}(0), L^{(2)} v_{1}(0)\right\rangle & +\left\langle\tilde{v}_{2}(0), L^{(1)} v_{2}(0)\right\rangle
\end{array}\right) .
$$


Yet, once proved, for $(j, l) \neq(1,2)$,

claim_prop

$$
\left\langle\partial_{\xi} \tilde{v}_{j}(0), v_{l}(0)\right\rangle=\left\langle\tilde{v}_{j}(0), \partial_{\xi} v_{l}(0)\right\rangle=0 \quad \text { and } \quad \partial_{\xi} v_{1}(0)=i \bar{k} \partial_{k} \bar{U}
$$

a direct examination of $\check{M}_{0}($ using $(3.7))$ shows

$$
\check{M}_{0}=\left[\begin{array}{cc}
-\bar{k} \partial_{k} \bar{c} & -\bar{k} \partial_{M} \bar{c} \\
-\partial_{k} \bar{F} & -\partial_{M} \bar{F}+\bar{c}
\end{array}\right] .
$$

Hence the result. We are left with the proof of $\left(\begin{array}{lc}\text { ( } l \text { laim_prop } \\ .55) \text {. }\end{array}\right.$

First, since expanding the duality relation $\left\langle\tilde{v}_{2}(\xi), v_{1}(\xi)\right\rangle=0$ yields $\left\langle\partial_{\xi} \tilde{v}_{2}(0), v_{1}(0)\right\rangle+$ $\left\langle\tilde{v}_{2}(0), \partial_{\xi} v_{1}(0)\right\rangle=0$, it is licit to replace $\tilde{v}_{2}(\xi)$ with $\tilde{v}_{2}(\xi)-\left\langle\partial_{\xi} \tilde{v}_{2}(0), v_{1}(0)\right\rangle \xi \tilde{v}_{1}(\xi)$ and $v_{1}(\xi)$ with $v_{1}(\xi)-\left\langle\tilde{v}_{2}(0), \partial_{\xi} v_{1}(0)\right\rangle \xi v_{2}(\xi)$. Thus we may assume the first part of the claim is satisfied when $(j, l)=(2,1)$. Now fix $l$ and normalize $v_{l}(\xi)$ according to $\left\langle\tilde{v}_{l}(0), v_{l}(\xi)\right\rangle=1$. This yields $\left\langle\tilde{v}_{l}(0), \partial_{\xi} v_{l}(0)\right\rangle=0$. Yet, expanding the duality relation $\left\langle\tilde{v}_{l}(\xi), v_{l}(\xi)\right\rangle=1$ at first order in $\xi$ gives $\left\langle\partial_{\xi} \tilde{v}_{l}(0), v_{l}(0)\right\rangle+\left\langle\tilde{v}_{l}(0), \partial_{\xi} v_{l}(0)\right\rangle=0$. From this the first part of the claim follows when $j=l$.

Now let us denote

$$
\Pi(\xi)=\left\langle\tilde{v}_{1}(\xi), \cdot\right\rangle v_{1}(\xi)+\left\langle\tilde{v}_{2}(\xi), \cdot\right\rangle v_{2}(\xi) .
$$

Expanding $L_{\xi} v_{1}(\xi)=\Pi(\xi) L_{\xi} v_{1}(\xi)$ at first order in $\xi$ and using $\left(\begin{array}{ll}\mathrm{dk} \\ 3.7\end{array}\right)$ provide

$$
L_{0}\left[\partial_{\xi} v_{1}(0)-i \bar{k} \partial_{k} \bar{U}\right]=\Pi(0) L_{0}\left[\partial_{\xi} v_{1}(0)-i \bar{k} \partial_{k} \bar{U}\right] .
$$

Therefore there exist $\alpha$ and $\beta$ such that

$$
L_{0}\left[\partial_{\xi} v_{1}(0)-i \bar{k} \partial_{k} \bar{U}\right]=\alpha v_{1}(0)+\beta v_{2}(0) .
$$

Since $\tilde{v}_{2}(0)$ lies in the left kernel of $L_{0}, \beta=0$. Normalizing parameterization according to $(\mathrm{N})$, we deduce

$$
\partial_{\xi} v_{1}(0)=i \bar{k} \partial_{k} \bar{U}+\frac{\alpha}{-\bar{k} \partial_{M} \bar{c}} \partial_{M} \bar{U}
$$

Then the first part of the claim implies $\alpha=0$. This finishes the proof.

Actually the previous proposition holds assuming (A) only instead of the stronger (A'). This requires to write the first-order Whitham's system in an intrinsic way, not depending on the choice of the parameterization. The main achievement of $[21]$ is precisely the proof that for general conservation laws a similar proposition holds assuming only the non-degeneracy of the Poincaré return map (in the sense of $(\mathrm{A})$ ). There it is proved by a direct inspection of an Evans function.

We now state some easy consequences of the previous statements. We start with necessary conditions for side-band stability. Again for the first part of the next corollary (A') may be replaced with (A) (see $[21]$ ). In the second part, we use a nonstandard terminology for Kawashima's conditions. The Kawashima's conditions are a set of conditions dealing 
with stability of constant states for quasilinear (degenerate) hyperbolic-parabolic systems (see $\left[\frac{16}{16}\right]$ ). Besides structure assumptions the key conditions are: there is a symmetrization of the hyperbolic part that makes the viscous part nonnegative (usually required via the existence of a dissipative convex entropy); no hyperbolic mode lies in the kernel of the viscous part. According to consequences of these conditions, we say that the weak Kawashima's condition is satisfied at a point if there the action of the viscous part on hyperbolic modes is nonpositive and that the strong Kawashima's condition holds if this action is negative. To make it explicit for the second-order Whitham's system let us complete the $\left(\left(k_{0}^{(1)}, M_{0}^{(1)}\right),\left(k_{0}^{(2)}, M_{0}^{(2)}\right)\right)$ appearing in theorem $\frac{\text { main }}{3.2}$ with a dual basis of left eigenvectors $\left(\left(\tilde{k}_{0}^{(1)}, \tilde{M}_{0}^{(1)}\right),\left(\tilde{k}_{0}^{(2)}, \tilde{M}_{0}^{(2)}\right)\right)$. Then the weak Kawashima's condition at $(\bar{k}, \bar{M})$ is written

$$
j=1,2, \quad \Re\left(\left\langle\left(\begin{array}{c}
\tilde{k}_{0}^{(j)} \\
\tilde{M}_{0}^{(j)}
\end{array}\right),\left[\begin{array}{cc}
\bar{d}_{1,1} & \bar{d}_{1,2} \\
\bar{d}_{2,1} & \bar{d}_{2,2}
\end{array}\right]\left(\begin{array}{c}
k_{0}^{(j)} \\
M_{0}^{(j)}
\end{array}\right)\right\rangle_{\mathbb{R}^{2}}\right) \geq 0
$$

and the strong condition reads

$$
j=1,2, \quad \Re\left(\left\langle\left(\begin{array}{c}
\tilde{k}_{0}^{(j)} \\
\tilde{M}_{0}^{(j)}
\end{array}\right),\left[\begin{array}{cc}
\bar{d}_{1,1} & \bar{d}_{1,2} \\
\bar{d}_{2,1} & \bar{d}_{2,2}
\end{array}\right]\left(\begin{array}{c}
k_{0}^{(j)} \\
M_{0}^{(j)}
\end{array}\right)\right\rangle_{\mathbb{R}^{2}}\right)>0 .
$$

Note that, if the first-order Whitham's system is strictly hyperbolic, the real parts may be omitted in the previous inequalities because the above scalar products are already real, and, even in the weakly hyperbolic case, we may choose eigenvectors to make them so.

Corollary 3.4. Assume ( $\left.A^{\prime}\right)$.

1. Assume the first-order Whitham's system is not weakly hyperbolic at $(\bar{k}, \bar{M})$.

Then there exists $\varepsilon \in] 0, \pi[$ such that, (for all $\xi$ such that $0<\xi<\varepsilon$ ) or (for all $\xi$ such that $-\varepsilon<\xi<0$ )

$$
\sigma\left(L_{\xi}\right) \bigcap\{\lambda \mid \Re(\lambda)>0\} \neq \emptyset .
$$

2. Assume the first-order Whitham's system is strictly hyperbolic, but the second-order Whitham's system violates at $(\bar{k}, \bar{M})$ the weak Kawashima's condition.

Then there exists $\varepsilon \in] 0, \pi[$ such that, for all $\xi$ such that $0<|\xi|<\varepsilon$,

$$
\sigma\left(L_{\xi}\right) \bigcap\{\lambda \mid \Re(\lambda)>0\} \neq \emptyset .
$$

Proof. Thanks to proposition 3 submain 3 the first condition tells $\lambda_{0,1} \notin \mathbb{R}$ and $\lambda_{0,2} \notin \mathbb{R}$. The result follows then from $(3.1)$.

Thanks to proposition 3.3 the second condition implies $\lambda_{0,1} \in \mathbb{R}, \lambda_{0,2} \in \mathbb{R}$ and (B) is satisfied. Thus through theorem $3_{3.2}$ the second condition also yields $\Re\left(\lambda_{1}^{\prime \prime}(0)\right)>0$ or

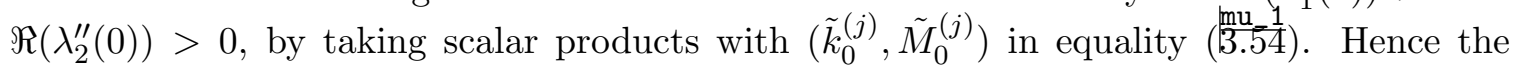
result. 
We now make precise the claim that strong co-periodic stability plus strong modulational stability yield side-band stability.

SB_stab Corollary 3.5. Assume (A') and the following conditions.

1. There exists $\eta>0$ such that

$$
\sigma\left(L_{0}\right) \subset\{0\} \cup\{\lambda \mid \Re(\lambda) \leq-\eta\}
$$

2. The first-order Whitham's system is strictly hyperbolic and the second-order Whitham's system satisfies the strong Kawashima's condition.

Then there exists $\varepsilon \in] 0, \pi[$ such that, for all $\xi$ such that $0<|\xi|<\varepsilon$,

$$
\sigma\left(L_{\xi}\right) \subset\{\lambda \mid \Re(\lambda)<0\} .
$$

Proof. Under these assumptions, (B) holds, $\Re\left(\lambda_{1}^{\prime}(0)\right)=0, \Re\left(\lambda_{2}^{\prime}(0)\right)=0, \Re\left(\lambda_{1}^{\prime \prime}(0)\right)<0$ and $\Re\left(\lambda_{2}^{\prime \prime}(0)\right)<0$. Therefore a perturbation argument provides the result.

We stop here the derivation of corollaries about spectral instability or stability criteria from our main results. Let us make a final comment about the fact that the coefficients of the second-order averaged system shares with those of many effective approximate systems whose derivation involves homogenization the unsatisfactory property that they are written in an intricate way. It may then seem that these criteria are of no practical unse. Yet let us mention that for the Kuramoto-Sivashinsky equation, the seminal work [11] already proposed a second-order modulation system, derived in a formal way and that therein a numerical study of the first and second-order necessary conditions for side-band stability was then performed. Up to the fact that such an approach does not capture all kinds of spectral instabilitiess, their conclusions are in good agreement with numerical studies of full stability [7, 2].

Besides stability criteria, the main motivation of theorem $\frac{\text { main }}{3.2}$ is the will to provide foundations for a nonlinear validation of the slow modulation ansatz and the corresponding second-order Whitham's system as a description of the asymptotic behavior near the wave. In this respect key relations are (3.52) and (3.51). Their particular role is the counterpart of the fact that many quasilinear hyperbolic-parabolic systems are asymptotically equivalent near constant states, and at the linear level their equivalence is read on the fact that they share the same hyperbolic modes and the same second-order low-frequency expansions for their eigenvalues (see [19]). Note that in the mentioned expansions for the second-order Whitham's system are read linear group velocities which are the characteristic speeds of the hyperbolic part $\left(\lambda_{0, j}-\bar{c}\right.$ with the above notations) and diffusion coefficients $\left(\left\langle\left(\begin{array}{c}\tilde{k}_{0}^{(j)} \\ \tilde{M}_{0}^{(j)}\end{array}\right),\left[\begin{array}{cc}\bar{d}_{1,1} & \bar{d}_{1,2} \\ \bar{d}_{2,1} & \bar{d}_{2,2}\end{array}\right]\left(\begin{array}{c}k_{0}^{(j)} \\ M_{0}^{(j)}\end{array}\right)\right\rangle_{\mathbb{R}^{2}}\right.$ with the above notations $)$. 


\section{sec4 4 The KdV limit}

Recall the authors' interest in the (KdV-KS) equation lies essentially in the $\delta$-small region. Yet a quick look at formulas of the previous section suggests that a result such as corollary 3.5 could only show stability for perturbations corresponding to Floquet parameters of size $o(\delta)$. Even at the formal level, something new is needed to try to capture the behavior corresponding to a full $(\xi, \delta)$-small region.

Our goal now is precisely to provide another modulation procedure and to infer from it stability conditions that are the natural candidates to decide stability in the full $(\xi, \delta)$-small region.

\section{Preliminaries}

The $\delta$-small limit is a singular perturbation of the KdV equation. Therefore the very first step of our discussion is to obtain a parameterization of a family of periodic traveling waves of $(\mathrm{KdV})$ providing for $(\mathrm{KdV})$ an analogous of condition $\left(\mathrm{A}^{\prime}\right)$. This may then be related to the structure of the generalized co-periodic kernels of the operator describing the $\mathrm{KdV}$ evolution linearized about a given wave (in a way similar to lemma $\frac{\pi}{2.1}$ ). One should also prove using geometric singular perturbation analysis that wave profiles of (KdV-KS) emerge smoothly from wave profiles of $(\mathrm{KdV})$. This is a nontrivial task as may be guessed from the fact that dimensions of respective sets of periodic traveling waves do not match.

As the focus of the present section is on the formal derivation, we do not formulate precise statements answering these questions. The reader is referred to [10] where is proved a wave profile perturbation result. One may also read [5] where the full Bloch spectrum of the $\mathrm{KdV}$ waves is investigated in details (including explicit resolvent formulas). This latter work relies heavily on the integrability of the KdV equation.

Actually the KdV waves we will work about are explicit, being cnoidal waves. Yet we restrain from giving explicit formulas and choose a parameterization convenient for the slow modulation discussion. We denote by $U_{\mathrm{cn}}(\cdot ; k, M, e)$ the solution, of period 1 , of

$$
k\left(3 U^{2}-c_{\mathrm{cn}}(k, M, e) U\right)^{\prime}+k^{3} U^{\prime \prime \prime}=0, \quad\langle U\rangle=M, \quad \frac{1}{2}\left\langle U^{2}\right\rangle=e .
$$

Waves of $(\mathrm{KdV})$ are recovered through $u(x, t)=U_{\mathrm{cn}}\left(k\left(x+\phi-c_{\mathrm{cn}}(k, M, e) t\right) ; k, M, e\right)$ (where $\phi$ is an arbitrary phase shift). As for (KdV-KS) arbitrary profiles may be recovered from zero-mean profiles since we can split profiles along

$$
U_{\mathrm{cn}}(\cdot ; k, M, e)=M+U_{\mathrm{cn}}^{(0)}\left(\cdot ; k, e-M^{2} / 2\right), \quad c_{\mathrm{cn}}(k, M, e)=6 M+c_{\mathrm{cn}}^{(0)}\left(k, e-M^{2} / 2\right) .
$$

On wave profiles for $(\mathrm{KdV}$-KS), we mark now the $\delta$-dependence. They expand as

$$
U^{\delta}(\cdot ; k, M)=U_{0}(\cdot ; k, M)+\mathcal{O}(\delta), \quad c^{\delta}(k, M)=c_{0}(k, M)+\mathcal{O}\left(\delta^{2}\right),
$$

where

$$
U_{0}(\cdot ; k, M)=U_{\mathrm{cn}}\left(\cdot ; k, M, M^{2} / 2+E(k)\right), \quad c_{0}(k, M)=c_{\mathrm{cn}}\left(k, M, M^{2} / 2+E(k)\right),
$$


where $E$ is some function described implicitly below $\frac{\text { EMR }}{[10]}$. Again note that not all $\mathrm{KdV}$ waves generate $\mathrm{KdV}$-KS waves, the relation $e=M^{2} / 2+E(k)$ implements this selection principle.

Fix some wave parameters $(\bar{k}, \bar{M}, \bar{e})$ (a priori $\bar{e}$ is arbitrary) corresponding to a wave $\bar{U}_{\text {cn }}$ with speed $\bar{c}_{\mathrm{cn}}$ and introduce the $\mathrm{KdV}$ operator

$$
\mathbb{L}[g]=-\bar{k}\left(\left(6 \bar{U}_{\mathrm{cn}}-\bar{c}_{\mathrm{cn}}\right) g\right)^{\prime}-\bar{k}^{3} g^{\prime \prime \prime} .
$$

Denote $\mathbb{L}_{\xi}$ the corresponding Bloch symbol, which expands as

$$
\mathbb{L}_{\xi}=\mathbb{L}_{0}+i \bar{k} \xi \mathbb{L}^{(1)}+(i \bar{k} \xi)^{2} \mathbb{L}^{(2)}+(i \bar{k} \xi)^{3} \mathbb{L}^{(3)}
$$

Then the value 0 is an eigenvalue of $\mathbb{L}_{0}$ of geometric multiplicity 2 and algebraic multiplicity 3. Moreover

$$
\mathbb{L}_{0} \bar{U}_{\mathrm{cn}}^{\prime}=0, \quad \mathbb{L}_{0} \partial_{M} \bar{U}_{\mathrm{cn}}=-\bar{k} \partial_{M} \bar{c}_{\mathrm{cn}} \bar{U}_{\mathrm{cn}}^{\prime}, \quad \mathbb{L}_{0} \partial_{e} \bar{U}_{\mathrm{cn}}=-\bar{k} \partial_{e} \bar{c}_{\mathrm{cn}} \bar{U}_{\mathrm{cn}}^{\prime}
$$

and

$$
\mathbb{L}_{0}^{*} 1=0, \quad \mathbb{L}_{0}^{*} \bar{U}_{\mathrm{cn}}=0,
$$

(where $\mathbb{L}_{0}^{*}$ denotes the formal adjoint of $\mathbb{L}_{0}$ ) with pairing relations $\left\langle\bar{U}_{\mathrm{cn}}^{\prime}\right\rangle=\left\langle\partial_{e} \bar{U}_{\mathrm{cn}}\right\rangle=0$, $\left\langle\bar{U}_{\mathrm{cn}}, \bar{U}_{\mathrm{cn}}^{\prime}\right\rangle=\left\langle\bar{U}_{\mathrm{cn}}, \partial_{M} \bar{U}_{\mathrm{cn}}\right\rangle=0,\left\langle\partial_{M} \bar{U}_{\mathrm{cn}}\right\rangle=1$ and $\left\langle\bar{U}_{\mathrm{cn}}, \partial_{e} \bar{U}_{\mathrm{cn}}\right\rangle=1$. Note also that

$$
\mathbb{L}_{0} \partial_{k} \bar{U}_{\mathrm{cn}}+\bar{k} \partial_{k} \bar{c}_{\mathrm{cn}} \bar{U}^{\prime}+\mathbb{L}^{(1)} \bar{U}_{\mathrm{cn}}^{\prime}=0
$$

Now fix some wave parameters $(\bar{k}, \bar{M})$. To discuss further the spectral problem we are considering, let us mark the $\delta$ dependence on the operator $L$,

$$
L^{\delta}[g]=-\bar{k}\left(\left(6 \bar{U}^{\delta}-\bar{c}^{\delta}\right) g\right)^{\prime}-\bar{k}^{3} g^{\prime \prime \prime}-\delta\left(\bar{k}^{2} g^{\prime \prime}+\bar{k}^{4} g^{\prime \prime \prime \prime}\right)
$$

Denote $L_{\xi}^{\delta}$ the corresponding Bloch symbol. Our focus is thus on the critical spectral problems

$$
L_{\xi}^{\delta} q=\lambda q
$$

when $(\delta, \xi)$ is small. There are two natural zones where to build about.

First, setting $\bar{e}=\bar{M}^{2} / 2+E(\bar{k}), L_{\xi}^{0}=\mathbb{L}_{\xi}$, the spectrum of $\mathbb{L}_{\xi}$ is known [BD], and we may indeed expect to expand the spectrum of $L_{\xi}^{\bar{\delta}|\xi|}$ when $(\xi, \bar{\delta})$ is small. A relevant first-order modulation system should there be the one of the KdV equation In this area formal expan-

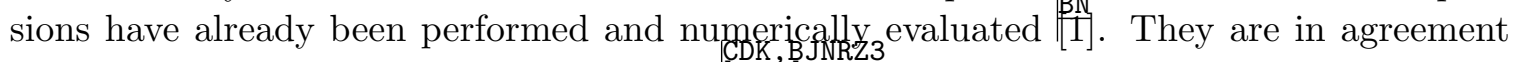
with full stability numerical investigations $[7,2]$.

Second, the spectrum of $L_{0}^{\delta}$ is known and we may also expect to expand the spectrum of $L_{\delta \bar{\xi}}^{\delta}$ when $(\bar{\xi}, \delta)$ is small. Here the relevant first-order modulation system is the limit when $\delta \rightarrow 0$ of the first-order Whitham's system of the previous section.

We insist on the fact that the dimensions of the two situations are different and that there is a priori no trivial way to relate them. Relying on a formal slow-modulation ansatz, we propose now intermediate modulation systems trying to fill this gap. They correspond to the situation where one fixes a $\bar{\delta}$ and expand the spectrum of $L_{\xi}^{\bar{\delta}|\xi|}$ in $\xi$ small. Then the limit $\bar{\delta} \rightarrow 0$ is related to the first zone and the singular limit $\bar{\delta} \rightarrow \infty$ brings us in the second area. 


\section{A modulation system}

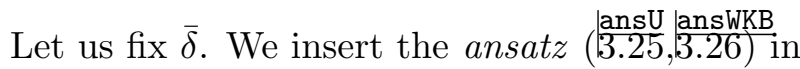

$$
\partial_{t} u+\partial_{x}\left(3 u^{2}\right)+\partial_{x}^{3} u+\bar{\delta} \varepsilon\left(\partial_{x}^{2} u+\partial_{x}^{4} u\right)=0 .
$$

First identification yields, with $\Omega_{0}=\partial_{T} \phi_{0}$ and $k_{0}=\partial_{X} \phi_{0}$,

meKdV_0 (4.2)

$$
\Omega_{0} \partial_{y} U_{0}+k_{0} \partial_{y}\left(3 U_{0}^{2}\right)+k_{0}^{3} \partial_{y}^{3} U_{0}=0
$$

solved with

$$
\begin{aligned}
\Omega_{0}(X, T) & =-k_{0}(X, T) c_{\mathrm{cn}}\left(k_{0}(X, T), M_{0}(X, T), e_{0}(X, T)\right) \\
U_{0}(y ; X, T) & =U_{\mathrm{cn}}\left(y, k_{0}(X, T), M_{0}(X, T), e_{0}(X, T)\right) .
\end{aligned}
$$

Next step is, setting $\Omega_{1}=\partial_{T} \phi_{1}$ and $k_{1}=\partial_{X} \phi_{1}$,

meKdV_1

$$
\begin{aligned}
\left(\Omega_{1}+c_{\mathrm{cn}, 0} k_{1}\right) U_{0}^{\prime} & -k_{1} \mathbb{L}^{(1)} U_{0}^{\prime}-\mathbb{L}_{0} U_{1}-\mathbb{L}^{(1)} \partial_{X} U_{0} \\
& -\partial_{X} k_{0} \mathbb{L}^{(2)} U_{0}^{\prime}+\partial_{T} U_{0}+c_{\mathrm{cn}, 0} \partial_{X} U_{0}=-\bar{\delta}\left(k_{0}^{2} U_{0}^{\prime \prime}+k_{0}^{4} U_{0}^{\prime \prime \prime \prime}\right) .
\end{aligned}
$$

Solvability conditions are obtained taking scalar products with 1 and $U_{0}$.

Compatibility equality $\partial_{T} k_{0}=\partial_{X} \Omega_{0}$, together with solvability conditions compose the modulation system

wKdV_0

$$
\left\{\begin{aligned}
\partial_{t} k_{0}+\partial_{x}\left(k_{0} c_{\mathrm{cn}}\left(k_{0}, M_{0}, e_{0}\right)\right) & =0 \\
\partial_{t} M_{0}+\partial_{x}\left(6 e_{0}\right) & =0 \\
\partial_{t} e_{0}+\partial_{x}\left(Q\left(k_{0}, M_{0}, e_{0}\right)\right) & =-\bar{\delta} R\left(k_{0}, M_{0}, e_{0}\right)
\end{aligned}\right.
$$

where we have used the following definitions, for a general $(\bar{k}, \bar{M}, \bar{e})$,

$$
Q(\bar{k}, \bar{M}, \bar{e})=2\left\langle\bar{U}^{3}\right\rangle-3 \bar{k} \bar{e}, \quad R(\bar{k}, \bar{M}, \bar{e})=\bar{k}^{4}\left\langle\left(\bar{U}^{\prime \prime}\right)^{2}\right\rangle-\bar{k}^{2}\left\langle\left(\bar{U}^{\prime}\right)^{2}\right\rangle .
$$

One may easily check that $R(\bar{k}, \bar{M}, \bar{e})=R\left(\bar{k}, 0, \bar{e}-\bar{M}^{2} / 2\right)$.

As a particular case, setting $\bar{\delta}=0$ recovers the first-order Whitham's system for KdV waves [25]. At the opposite, $\mathrm{KdV}-\mathrm{KS}$ waves correspond to constant solutions in the limit $\bar{\delta} \rightarrow \infty$. Therefore one reads on $\left(\frac{w K}{4.5}\right)$ the above mentioned selection principle

$$
R(\bar{k}, \bar{M}, \bar{e})=0,
$$

also written $\bar{e}=\bar{M}^{2} / 2+E(\bar{k})$. Going further one may look at the relaxed version of system $\left(\frac{w K}{4.5}\right)$

$$
\left\{\begin{aligned}
\partial_{t} k_{0}+\partial_{x}\left(k_{0} c_{\mathrm{cn}}\left(k_{0}, M_{0}, M_{0}^{2} / 2+E\left(k_{0}\right)\right)\right) & =0 \\
\partial_{t} M_{0}+\partial_{x}\left(3 M_{0}^{2}+6 E\left(k_{0}\right)\right) & =0
\end{aligned}\right.
$$

which coincides with the limit $\delta \rightarrow 0$ of system $\left(\frac{w 12}{3.34}\right)^{0}$. 


\section{Subcharacteristic conditions}

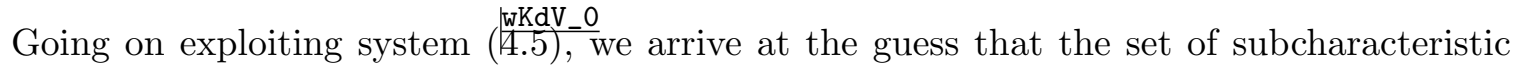
conditions

(S1) The system without relaxation $\left(\left(\frac{\mathrm{WKdV}-0}{4.5}\right)^{\text {with }} \bar{\delta}=0\right)$ is strictly hyperbolic.

(S2) Relaxation is dissipative, that is $\partial_{e} R\left(\bar{k}, \bar{M}, \bar{M}^{2} / 2+E(\bar{k})\right)>0$.

(S3) The relaxed system ( $(\text { W.6 })_{-}$is strictly hyperbolic.

(S4) Previous characteristic speeds intertwine, that is, ordering characteristic speeds of $\left(\frac{W}{4.5}\right)^{-}$with $\bar{\delta}=0$ as $\alpha_{1}<\alpha_{2}<\alpha_{3}$ and characteristic speeds of $\left(\frac{\text { WKd }}{4.6}\right)$ as $\beta_{1}<\beta_{2}$, is required $\alpha_{1}<\beta_{1}<\alpha_{2}<\beta_{2}<\alpha_{3}$.

is a natural candidate to provide sufficient conditions for side-band stability uniformly in $\delta$ small. Replacing strictly with weakly and strict inequalities with large inequalities gives a natural candidate for necessary conditions.

It may seem that these conditions are far from considerations on the Kawashima's condition of the previous section. It is not so, first the subcharacteristic conditions are a reformulation of the Kawashima's condition for (degenerate) quasilinear hyperbolic systems with relaxation; second, relaxation limit theory also provides a viscous correction to $\left(\frac{\text { Whd }}{4.6}\right)$ via Chapman-Enskog expansions. On this second-order modulation system one could read second-order terms for eigenvalues expansions, and the fact that under subcharacteristic

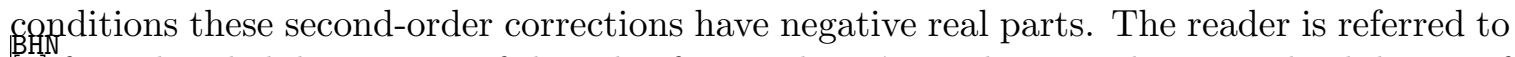
4] for a detailed description of the role of Kawashima's condition and a general validation of Chapman-Enskog expansion in the context of (degenerate) quasilinear hyperbolic systems with relaxation.

\section{Conclusion}

We studied the prescription power of modulation averaged systems on spectral stability of periodic wave trains. For its own interest and its canonical nature, we focused on the Korteweg-de Vries-Kuramoto-Sivashinsky equation. Yet, except for the KdV limit part, the arguments are both general and robust.

We have first shown how to obtain modulation averaged equations approximating evolution of local parameters in a slow modulation regime. Then we have related eigenmodes of the original equation with those of the modulation systems in the respective low-Floquet and low-Fourier regimes. Relation on eigenvalues are readily converted into stability criteria, whereas we hope that relations on eigenfunctions and eigenvectors could serve as the main spectral lemma in a nonlinear justification of the slow modulation ansatz. Note that numerical studies show that spectrally stable wave trains (in the diffusive sense discussed here) do exist [7, 2], and this spectral stability may be converted in a nonlinear stability under localized perturbations $\frac{3}{2]}$. However up to now nonlinear results are not precise enough to validate the full modulation scenario. 
The universal nature of (KdV-KS) stems from the fact that it can be derived in a $\delta \rightarrow 0$ regime from many similar situations. Yet expansions discussed previously are not uniform with respect to $\delta$. In the last part of the paper, we tried to explain how to fix this in the slow modulation ansatz. This revealed some subcharacteristic conditions we believe crucial for understanding the side-band stability in the $\delta \rightarrow 0$ limit. We do not expect to also deduce from modulation theory expansion of eigenfunctions uniformly in $\delta$. Yet there is some hope to prove that modulation predictions are again correct at the eigenvalue level by working on dispersion relations, either from Evans functions (as in [21]) or from critical Bloch matrices (as in proofs of lemma 3.1 and proposition 3.3). Since the spectrum of periodic traveling waves of $(\mathrm{KdV})$ is explicitly known $[5]$, we even expect to be able to combine low-Floquet arguments validating the subcharacteristic conditions, with highFloquet energy estimates and examination of regular expansions of intermediate-Floquet eigenvalues in order to obtain for small $\delta$ an analytic proof of diffusive spectral stability for a band of periodic traveling waves of (KdV-KS).

\section{References}

BN [1] Doron E. Bar and Alexander A. Nepomnyashchy. Stability of periodic waves governed by the modified Kawahara equation. Phys. D, 86(4):586-602, 1995.

BJNRZ3 [2] Blake Barker, Mathew A. Johnson, Pascal Noble, L. Miguel Rodrigues, and Kevin Zumbrun. Non linear modulational stability of periodic traveling-wave solutions of the generalized kuramoto-sivashinsky equation. In preparation.

BJNRZ4 [3] Blake Barker, Mathew A. Johnson, Pascal Noble, L. Miguel Rodrigues, and Kevin Zumbrun. Stability of periodic kuramoto-sivashinsky waves. Applied Mathematics Letters. To appear.

BHN [4] Stefano Bianchini, Bernard Hanouzet, and Roberto Natalini. Asymptotic behavior of smooth solutions for partially dissipative hyperbolic systems with a convex entropy. Comm. Pure Appl. Math., 60(11):1559-1622, 2007.

BD [5] Nate Bottman and Bernard Deconinck. KdV cnoidal waves are spectrally stable. Discrete Contin. Dyn. Syst., 25(4):1163-1180, 2009.

[6] Hsueh-Chia Chang and Evgeny A. Demekhin. Complex wave dynamics on thin films, volume 14 of Studies in Interface Science. Elsevier Science B.V., Amsterdam, 2002.

$\mathrm{CDK}$

[7] Hsueh-Chia Chang, Evgeny A. Demekhin, and Dmitry I. Kopelevich. Laminarizing effects of dispersion in an active-dissipative nonlinear medium. Phys. D, 63:299-320, 1993.

[8] Michael C. Cross and Pierre C. Hohenberg. Pattern formation out of equilibrium. Rev. Mod. Phys., 65:851-1112, 1993. 
REFERENCES

D3S [9] Arjen Doelman, Björn Sandstede, Arnd Scheel, and Guido Schneider. The dynamics of modulated wave trains. Mem. Amer. Math. Soc., 199(934):viii+105, 2009.

EMR [10] Nicholas M. Ercolani, David W. McLaughlin, and Heinz Roitner. Attractors and transients for a perturbed periodic KdV equation: a nonlinear spectral analysis. J. Nonlinear Sci., 3(4):477-539, 1993.

FST [11] Uriel Frisch, Zhen-Su She, and Olivier Thual. Viscoelastic behaviour of cellular solutions to the Kuramoto-Sivashinsky model. J. Fluid Mech., 168:221-240, 1986.

G [12] Robert A. Gardner. On the structure of the spectra of periodic travelling waves. J. Math. Pures Appl. (9), 72(5):415-439, 1993.

JZ1 [13] Mathew A. Johnson and Kevin Zumbrun. Nonlinear stability of periodic traveling wave solutions of systems of viscous conservation laws in the generic case. J. Differential Equations, 249(5):1213-1240, 2010.

Ka [14] Tosio Kato. Perturbation theory for linear operators. Classics in Mathematics. SpringerVerlag, Berlin, 1995. Reprint of the 1980 edition.

KaTo [15] Takuji Kawahara and Sadayoshi Toh. Pulse interactions in an unstable dissipativedispersive nonlinear system. Phys. Fluids, 31(8):2103-2111, 1988.

Kaw [16] Schuichi Kawashima. Systems of a hyperbolic-parabolic composite type, with applications to the equations of magnetohydrodynamics. PhD thesis, Kyoto University, 1983.

K [17] Yoshiki Kuramoto. Chemical oscillations, waves, and turbulence, volume 19 of Springer Series in Synergetics. Springer-Verlag, Berlin, 1984.

KT [18] Yoshiki Kuramoto and Toshio Tsuzuki. On the formation of dissipative structures in reaction-diffusion systems. Progress of Theoretical Physics, 54(3), 1975.

LZ [19] Tai-Ping Liu and Yanni Zeng. Large time behavior of solutions for general quasilinear hyperbolic-parabolic systems of conservation laws. Mem. Amer. Math. Soc., 125(599):viii+120, 1997.

PSU [20] Robert L. Pego, Guido Schneider, and Hannes Uecker. Long-time persistence of Korteweg-de Vries solitons as transient dynamics in a model of inclined film flow. Proc. Roy. Soc. Edinburgh Sect. A, 137(1):133-146, 2007.

Se [21] Denis Serre. Spectral stability of periodic solutions of viscous conservation laws: large wavelength analysis. Comm. Partial Differential Equations, 30(1-3):259-282, 2005.

S1 [22] Gregory I. Sivashinsky. Nonlinear analysis of hydrodynamic instability in laminar flames. I. Derivation of basic equations. Acta Astronaut., 4(11-12):1177-1206, 1977.

S2 [23] Gregory I. Sivashinsky. Instabilities, pattern formation, and turbulence in flames. Annual Review of Fluid Mechanics, 15(179-199):1177-1206, Jan. 1983. 
SM [24] Gregory I. Sivashinsky and D. M. Michelson. On irregular wavy flow of a liquid film down a vertical plane. Progress of Theoretical Physics, 63(6):2112-2114, 1980.

W [25] Gerald B. Whitham. Linear and nonlinear waves. Wiley-Interscience [John Wiley \& Sons], New York, 1974. Pure and Applied Mathematics.

Win [26] Htay Aung Win. Model equation of surface waves of viscous fluid down an inclined plane. J. Math. Kyoto Univ., 33(3):803-824, 1993.

Yu_Yang [27] Jun Yu and Yi Yang. Evolution of small periodic disturbances into roll waves in channel flow with internal dissipation. Stud. Appl. Math., 111(1):1-27, 2003. 